Energy Systems Environmental Restoration Program

ORNL Environmental Restoration Program

\title{
Waste Management Plan for the Remedial Investigation of Waste Area Grouping 2 at Oak Ridge National Laboratory, Oak Ridge, Tennessee,
}

Date Issued- October 1994

\author{
Prepared by \\ Environmental Sciences Division \\ Oak Ridge National Laboratory
}

Prepared for

U.S. Department of Energy

Office of Environmental Restoration and Waste Management under budget and reporting code EW 20

Environmental Restoration and Waste Management Programs

Oak Ridge National Laboratory

Oak Ridge, Tennessee 37831-6285 managed by

MARTIN MARIETTA ENERGY SYSTEMS, INC.

for the

U.S. DEPARTMENT OF ENERGY

under contract DE-AC05-84OR21400 


\section{DISCLAIMER}

This report was prepared as an account of work sponsored by an agency of the United States Government. Neither the United States Government nor any agency Thereof, nor any of their employees, makes any warranty, express or implied, or assumes any legal liability or responsibility for the accuracy, completeness, or usefulness of any information, apparatus, product, or process disclosed, or represents that its use would not infringe privately owned rights. Reference herein to any specific commercial product, process, or service by trade name, trademark, manufacturer, or otherwise does not necessarily constitute or imply its endorsement, recommendation, or favoring by the United States Government or any agency thereof. The views and opinions of authors expressed herein do not necessarily state or reflect those of the United States Government or any agency thereof. 


\section{DISCLAIMER}

Portions of this document may be illegible in electronic image products. Images are produced from the best available original document. 


\section{Author Affiliation}

L.A. Baron is affiliated with the Environmental Sciences Division through subcontract with JAYCOR. This organization is managed by Martin Marietta Energy Systems, Inc. 
Waste Management Plan for the Remedial Investigation of Waste Area Grouping 2 at Oak Ridge National Laboratory, Oak Ridge, Tennessee

ORNL/ER-235

APPROVALS

ER-12-1505 (Effective 09-29-94)

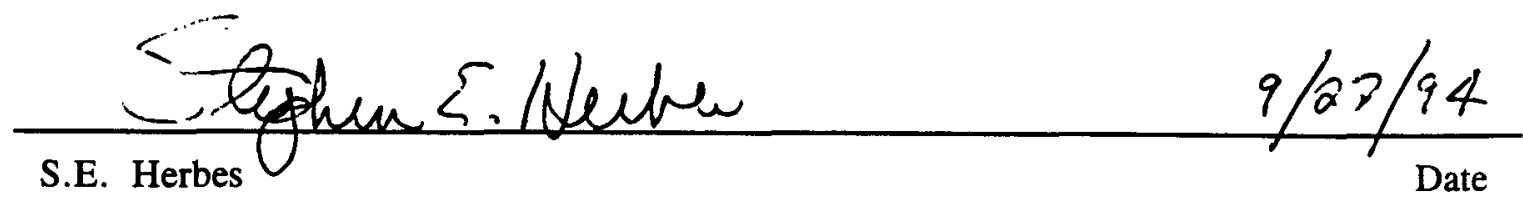

Project Manager

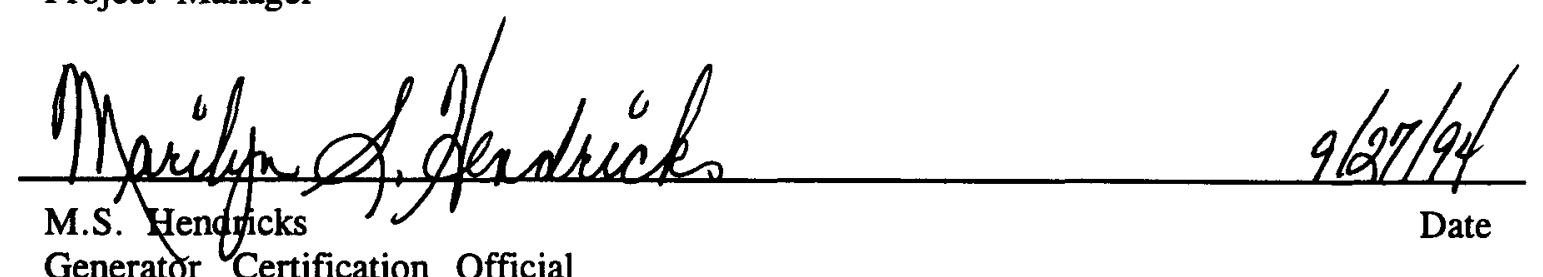

Generator Certification Official

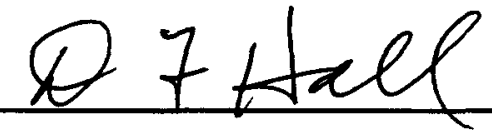

D.F. Hall

ORNL Waste Management Operation Organization

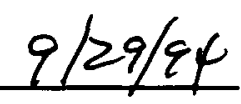

Date



C. Clark, Jr.

ORNL Environmental Restoration Program

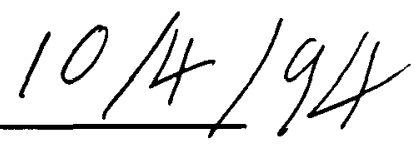

Date

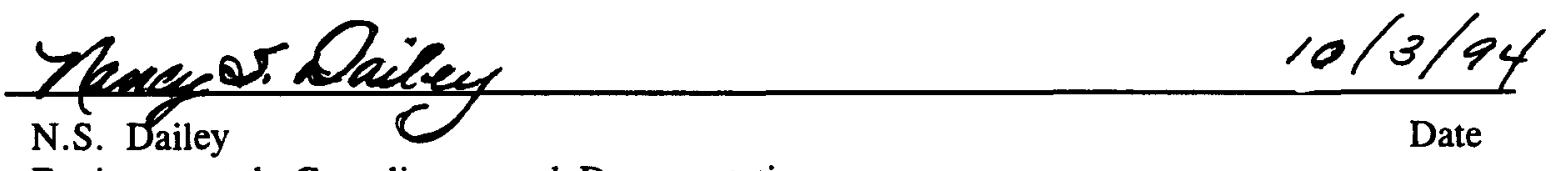

Environmental Compliance and Documentation

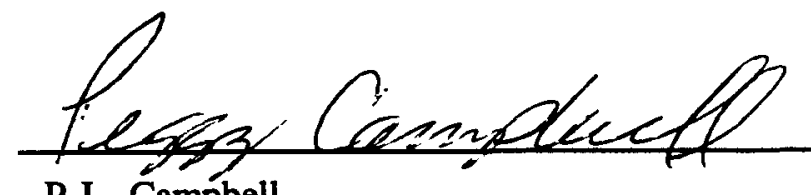

P.J. Campbell

Laboratory Certification Official 



\section{CONTENTS}

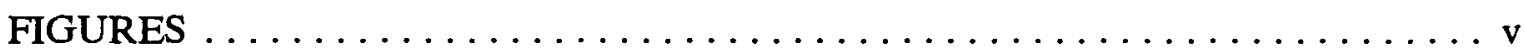

TABLES $\ldots \ldots \ldots \ldots \ldots \ldots \ldots \ldots \ldots \ldots \ldots \ldots \ldots \ldots \ldots \ldots \ldots \ldots \ldots$

ABBREVIATIONS $\ldots \ldots \ldots \ldots \ldots \ldots \ldots \ldots \ldots \ldots \ldots \ldots \ldots \ldots \ldots \ldots \ldots \ldots$ vii

EXECUTTVE SUMMARY $\ldots \ldots \ldots \ldots \ldots \ldots \ldots \ldots \ldots \ldots \ldots \ldots \ldots \ldots$ ix



1.1 REGULATORY OVERVIEW $\ldots \ldots \ldots \ldots \ldots \ldots \ldots \ldots \ldots \ldots \ldots \ldots \ldots \ldots \ldots \ldots$

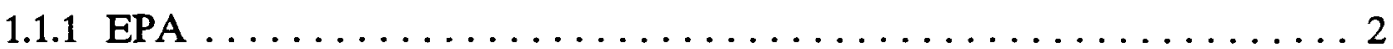

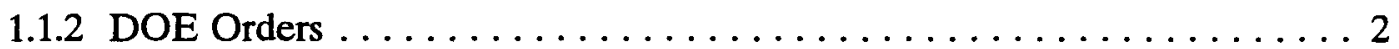

1.1.3 Occupational Safety and Health Administration $\ldots \ldots \ldots \ldots \ldots \ldots$

1.1.4 DOE Radiological Control Manual ................. 3

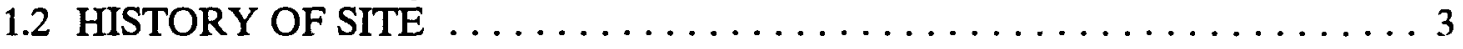

1.3 PURPOSE AND SCOPE OF ACTIVITY $\ldots \ldots \ldots \ldots \ldots \ldots \ldots \ldots$

2. LINE OF RESPONSIBILITIES $\ldots \ldots \ldots \ldots \ldots \ldots \ldots \ldots \ldots \ldots \ldots \ldots \ldots \ldots \ldots \ldots$

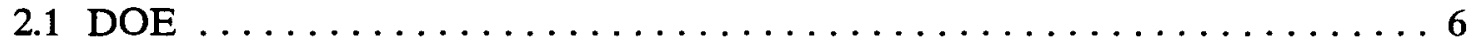

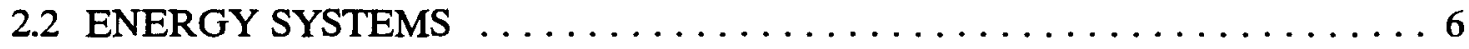

2.2 .1 Project Manager . . . . . . . . . . . . . . . . . . 6

2.2.2 Energy Systems Waste Management Organization ........... 7

2.2.3 Energy Systems ORNL Waste Management Organization ......... 7

2.2.4 ORNL Environmental Compliance Organization . . . . . . . . . 7

2.2.5 Environmental Restoration Central Waste Management Organization ... . 7

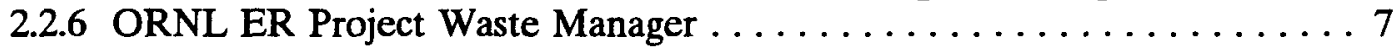

2.2 .7 Generator Certification Official ................... 8

2.2 .8 Laboratory Certification Official $\ldots \ldots \ldots \ldots \ldots \ldots \ldots \ldots \ldots \ldots$

2.2 .9 Generator . . . . . . . . . . . . . . . . . . . . . 9

2.2.10 Radioactive Solid Waste Operations Group . . . . . . . . . . 10

2.2.11 Hazardous Waste Operation Group $\ldots \ldots \ldots \ldots \ldots \ldots \ldots \ldots$



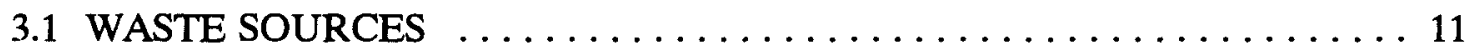

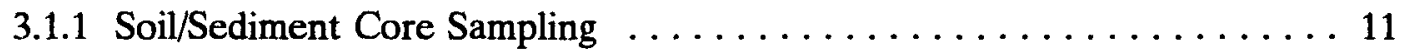

3.1.2 Developing and/or Purging Monitoring Wells $\ldots \ldots \ldots \ldots \ldots \ldots \ldots 12$



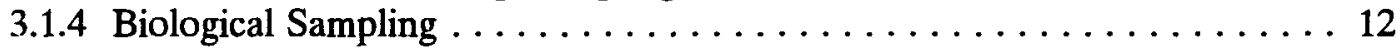

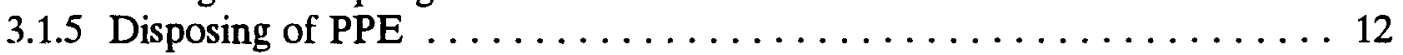

3.1.6 Decontaminating Small Tools By Hand Techniques . . . . . . . . 12

3.1.7 Sample Residual From Analytical Laboratory .............. 13

4. TYPES AND VOLUME OF WASTE $\ldots \ldots \ldots \ldots \ldots \ldots \ldots \ldots \ldots \ldots \ldots \ldots \ldots$ 
5. PROCEDURES FOR MANAGING WASTE

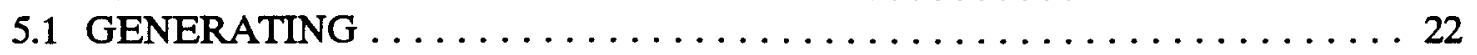

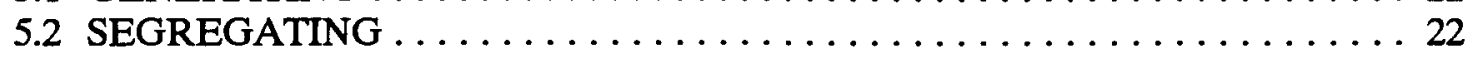

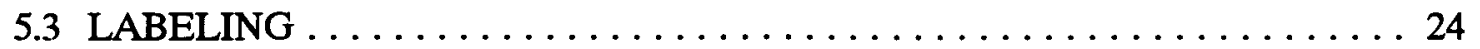

5.4 WASTE PACKAGING. HANDLING. AND DISPOSAL $\ldots \ldots \ldots \ldots \ldots 25$ 


\section{ABBREVIATIONS}

$\begin{array}{ll}\text { ALARA } & \text { as low as reasonably achievable } \\ \text { ARARs } & \text { relevant and appropriate requirements } \\ \text { C-zone } & \text { contamination zone coverall } \\ \text { CERCLA } & \text { Comprehensive Environmental Response, Compensation, and } \\ & \text { Liability Act } \\ \text { DCG } & \text { Derived Concentration Guide } \\ \text { DOE } & \text { U.S. Department of Energy } \\ \text { DOT } & \text { U.S. Department of Transportation } \\ \text { Energy Systems } & \text { Martin Marietta Energy Systems, Inc. } \\ \text { EPA } & \text { U.S. Environmental Protection Agency } \\ \text { EPO } & \text { Environmental Protection Officer } \\ \text { ER } & \text { Environmental Restoration } \\ \text { ESWMO } & \text { Energy Systems Waste Management Organization } \\ \text { GCO } & \text { Generator Certification Official } \\ \text { ES\&H } & \text { environmental, safety, and health } \\ \text { ESD } & \text { Environmental Sciences Division } \\ \text { FS } & \text { feasibility study } \\ \text { IDW } & \text { investigation-derived waste } \\ \text { LCO } & \text { Laboratory Certification Official } \\ \text { NRWTP } & \text { Nonradiological Wastewater Treatment Plant } \\ \text { ORNL } & \text { Oak Ridge National Laboratory } \\ \text { OSHA } & \text { Occupational Safety and Health Administration } \\ \text { PCB } & \text { polychlorinated biphenyl } \\ \text { PEL } & \text { permissible exposure limit } \\ \text { PPE } & \text { personal protective equipment } \\ \text { PWMP } & \text { project waste management plan } \\ \text { PWTP } & \text { process waste treatment plant } \\ \text { QA } & \text { quality assurance } \\ \text { RCRA } & \text { Resource Conservation and Recovery Act } \\ \text { RI } & \text { remedial investigation } \\ \text { RFDs } & \text { Request for Disposal Forms } \\ \text { RTR } & \text { real-time radiography } \\ \text { RSWO } & \text { Radioactive Solid Waste Operations } \\ \text { SARA } & \text { Superfund Amendments and Reauthorization Act } \\ \text { SHSO } & \text { Site Health and Safety Officer } \\ \text { TCLP } & \text { toxicity characteristic leaching procedure } \\ \text { TDEC } & \text { Tennessee Department of Environment and Conservation } \\ \text { TLV } & \text { threshold limit value } \\ \text { TOC } & \text { total organic carbon } \\ \text { TSS } & \text { total suspended solids } \\ \text { TTO } & \text { total toxic organics } \\ \text { VLA } & \text { very low activity waste } \\ \text { WAG } & \text { waste area grouping } \\ \text { WMO } & \text { Waste Management Operations } \\ & \\ & \\ & \end{array}$

vii 
WMRAD

WOC

WOCE

WOL
Waste Management and Remedial Action Division White Oak Creek

White Oak Creek Embayment

White Oak Lake 


\section{EXECUTIVE SUMMARY}

This Project Waste Management Plan defines the criteria and methods to be used for managing waste generated during activities associated with Waste Area Grouping 2 at Oak Ridge National Laboratory. The waste management strategy is based on the generation and management of waste on a systematic basis using the most appropriate combination of waste reduction, segregation, treatment, storage, and disposal practices while protecting the environment and human health, maintaining as low as reasonably achievable limits.

This plan contains provisions for safely and effectively managing soils and sediments, sampling water, decontamination fluids, and disposable personal protective equipment consistent with the U.S. Environmental Protection Agency guidance. This plan will be used in conjunction with the ORNL ER Program Waste Management Plan. 


\section{INTRODUCTION}

This Project Waste Management Plan (PWMP) will be used in conjunction with the ORNL ER Program Waste Management Plan. This PWMP defines the criteria and methods to be used for managing waste generated during activities associated with Waste Area Grouping (WAG) 2 at Oak Ridge National Laboratory (ORNL). The waste management strategy is based on the generation and management of waste on a systematic basis using the most appropriate combination of waste reduction, segregation, treatment, storage, and disposal practices while protecting the environment and human health, maintaining as low as reasonably achievable (ALARA) limits.

This plan contains provisions for safely and effectively managing soils and sediments, sampling water, decontamination fluids, and disposable personal protective equipment (PPE) consistent with the U.S. Environmental Protection Agency (EPA) guidance in Management of Investigation-Derived Waste During Site Investigation (EPA 1991) and best management practices. Consistent with EPA guidance, this plan is designed to protect the environment and the health and safety of workers and the public. It therefore specifies that investigationderived waste (IDW) and other wastes be managed to ensure that

- costs associated with sample storage, analysis, transportation, and disposal are minimized;

- additional pathways of contamination release are not created, and existing pathways are not enhanced;

- additional contaminated areas are not created; and

- potential worker exposures are not increased during the investigation and during the period before, during, and after remedial action.

The intent of this plan is to provide guidance for waste handling and disposal using both field screening (i.e., portable survey equipment) and media sampling and analysis in support of transferring waste to Martin Marietta Energy Systems, Inc. (Energy Systems), Waste Management Operations.

The provisions of this plan will be implemented through project procedures and other Energy Systems directives to ensure consistent waste management operations.

\subsection{REGULATORY OVERVIEW}

This plan was developed in accordance with published regulatory standards that are used as compliance documents at ORNL and other U.S. Department of Energy- (DOE-) owned or DOE-controlled sites. It incorporates elements of the standards and procedures that exhibit best management practices and guidelines for managing radioactive, mixed, and Resource Conservation and Recovery Act (RCRA) defined materials. 


\subsubsection{EPA}

Management of Investigation-Derived Wastes During Site Investigations, EPA/540/G-91/009, (EPA 1991), provides direction for managing IDW under the Comprehensive Environmental Response, Compensation, and Liability Act (CERCLA). This guidance is consistent with the applicable or relevant and appropriate requirements (ARARs) normally applied to remedial investigation/feasibility study (RI/FS) activities.

The guide requires that management techniques protect human health and the environment and comply with (or obtain waivers of) ARARs. The EPA guide states that RI/FS and remedial design actions must comply with ARARs "to the extent practicable considering the exigencies of the situation" (EPA 1990). This document is applicable to field activities associated with the ORNL RI/FS Project. It is presumed that adherence to the provisions of this guidance document will satisfy the requirements of CERCLA, RCRA, the Hazardous Solid Waste Amendments of 1984, and the Emergency Planning and Community Right-to-Know Act as they apply to IDW and other wastes generated by the ORNL RI/FS Project.

\subsubsection{DOE Orders}

DOE Order 5820.2A, Radioactive Waste Management, governs the handling and disposal of radioactive materials resulting from DOE activities. This order specifies four performance criteria that must be met in the management and disposal of low-level radioactive wastes: (1) protect public health and safety in accordance with standards specified in applicable Environmental Health Orders and other DOE orders; (2) ensure that exposures resulting from all credible pathways result in an effective dose equivalents that do not exceed $25 \mathrm{mrem} / \mathrm{year}$ to any member of the public and that releases to the atmosphere shall meet the requirements of 40 Code of Federal Regulations (CFR) 61 (EPA 1988); (3) ensure that the dose to an inadvertent intruder into the facility after loss of institutional controls (100 years) will not exceed $100 \mathrm{mrem} /$ year for continuous exposure or $500 \mathrm{mrem} / \mathrm{year}$ for a single acute exposure; and (4) protect groundwater resources consistent with federal, state, and local requirements (DOE 1988a). This order is consistent with EPA guidance in specifying that actions taken with radioactive wastes must protect human health and the environment.

DOE Order 5480.11, Radiation Protection for Occupational Workers, establishes a regulatory limit of 5 rem/year for the combined internal and external exposures of occupational workers to ionizing radiation and radioactive materials (DOE 1988b). The order also calls for the establishment of ALARA goals and objectives for each facility and operation commensurate with its mission. The ALARA philosophy has been subsequently incorporated into ORNL Health Physics Procedure RP-3.1 (ORNL 1992a) in the form of an administrative limit of 2 rem/year. DOE Order 5480.11 has been revised to require implementation of the DOE Radiological Control Manual (see Sect. 1.1.4).

DOE Order 5400.5, Radiation Protection of the Public and the Environment, establishes standards and requirements for DOE activities to protect the public and the environment against undue risk from radiation. This order presents the derived concentration guides for radioactive material in air and water that will expose an individual to $100 \mathrm{mrem}$ of annual effective dose equivalent under continuous exposure conditions. Chapter 4, "Residual Radioactive Material", presents radiological protection requirements and guidelines for 
cleanup of residual radioactive material, management of the resulting wastes and residues, and release of property. It is the objective of this order that potential exposures to members of the public and workers be maintained ALARA (DOE 1990).

\subsubsection{Occupational Safety and Health Administration (29 CFR 1910)}

The Occupational Safety and Health Administration (OSHA) has established standards for limiting occupational exposures to hazardous materials. Presented in 29 CFR 1910 is a range of standards applicable to RI/FS activities and other ORNL operations. Subpart 120, which governs hazardous waste operations, and Subpart $Z$, which establishes the permissible exposure limits (PELs) for occupational exposure to listed hazardous chemical agents, apply to waste management operations involving IDW (OSHA 1991).

The requirements of 29 CFR 1910 are incorporated in the ORNL Industrial Hygiene Procedures and Policies (ORNL 1992b) and the RI/FS Environmental, Safety, and Health $(E S \& H)$ Project Procedures. Because the PEL list in not comprehensive, the procedures permit the use of the American Conference of Governmental Industrial Hygienists threshold limit values (TLVs) as standards (ACGIH 1991). TLVs are used during field operations when they are more conservative than PELs.

\subsubsection{DOE Radiological Control Manual (DOE N 5480.6)}

By order of the Secretary of Energy in December 1991, selected elements of DOE Headquarters Environmental Health and Nuclear Energy organizations were directed to develop guidance for uniform health physics practices among all DOE operation sites; in June 1992, DOE published the Radiological Control Manual. All DOE maintenance and operations contractors were directed by the Secretary to prepare an implementation strategy by August 1992, to issue site-specific radiological control manuals by December 1992, and to complete all required standardized DOE Core Radiological Controls training and qualification upgrades by June 1994 (DOE 1992).

\subsection{HISTORY OF SITE}

WAG 2 consists of White Oak Creek (WOC) and its tributaries downstream of the main ORNL site, White Oak Lake (WOL), White Oak Creek Embayment (WOCE) on the Clinch River, and the associated floodplain and subsurface environment (Fig. 1). The WOC system is the surface drainage for the major ORNL WAGs and has been exposed to a diversity of contaminants from operations and waste disposal activities in the WOC watershed. WAG 2 therefore acts as a conduit through which hydrologic fluxes carry contaminants from upgradient areas to the Clinch River. Water, sediment, soil, and biota in WAG 2 are contaminated and continue to receive contaminants from upgradient WAGs.

The contaminants found throughout the WOC watershed are primarily a result of radioactive effluents that have been released from ORNL for almost 5 decades. Contaminants of concern found in surface water are Tritium (H-3), Strontium (Sr-90), polychlorinated biphenyls (PCBs), and possibly some trace metals (e.g., As, $\mathrm{Ba}, \mathrm{Cr}, \mathrm{Hg}, \mathrm{Se}$ ). Aquatic sediments and floodplain soils are the primary pools for contaminants including radionuclides such as Cesium (Cs-137) and Cobalt (Co-60), metals, and organic compounds. A detailed history of the WOC system can be found in the WAG 2 Remedial Investigation Plan (1990), 




Fig. 1 Location of waste area groupings (WAGs) and WAG 2 boundary. 
including detailed descriptions of specific sampling tasks.

\subsection{PURPOSE AND SCOPE OF ACTIVITY}

The purpose of the RI of WAG 2 is based on a long-term sampling and analysis strategy aimed at four key goals

1. implement, in concert with other programs, long-term multimedia environmental monitoring and tracking of contaminants leaving other WAGs, entering WAG 2, and being transported off-site;

2. provide a conceptual framework to integrate and develop information at the watershedlevel for pathways and processes that are key to contaminant movement, and so support remedial efforts at ORNL;

3. provide periodic updates of estimates of potential risk (both human health and ecological) associated with contaminants accumulating in and moving through WAG 2 to off-site areas; and

4. support the ORNL ER Program efforts to prioritize, remediate, and verify remedial effectiveness for contaminated sites at ORNL through long-term monitoring and continually updated risk assessments.

Remedial investigations and remediations are either under way or are planned for contaminated areas hydrologically upgradient of WAG 2 . Therefore, contaminant inputs will change as individual upgradient areas are remediated and as natural decay processes occur. Because of this, implementation of corrective measures in WAG 2 will likely follow the completion of remediation of upgradient WAGs. A phased approach for the remedial investigation of WAG 2 has been initiated.

WAG 2 is complex and dynamic with diverse sources of contaminants and fluxes driven by changing environmental conditions. Rather than an exhaustive site characterization for all contaminants, media, and flow pathways, WAG 2 personnel will (1) focus on contaminants and pathways of greatest concern and (2) monitor and gather sufficient information for processes controlling or driving contaminant fluxes to construct an appropriate conceptual model for contaminant source areas and fluxes in WAG 2. This approach allows the early identification of remedial alternatives and focuses efforts on the gathering of data useful for evaluating alternatives to reduce risk (Miller 1993).

The multimedia environmental monitoring and characterization of WAG 2 includes the sampling and analysis of surface water, groundwater, sediment, floodplain soil, and biota. Tasks are defined in detail in the Sampling and Analysis Plan for Waste Area Grouping 2 at Oak Ridge National Laboratory, Oak Ridge, Tennessee (ORNL 1992). 


\section{LINE OF RESPONSIBILITIES}

The WAG 2 RI project will be accomplished with the joint resources of the following participants:

1. DOE

2. Energy Systems (facility manager)

3. WAG 2 personnel subcontractors

Details of the organizational structure for implementation of this project, key personnel and their project responsibilities are as defined in the Remedial Investigation Plan for Waste Area Grouping 2 (ORNL 1990). For the purpose of this PWMP, a brief summary of responsibilities and duties is described in the following subsections.

\subsection{DOE}

DOE will furnish overall program management and provide project support as required through the DOE ORNL site office. Additionally, all coordination with EPA and the Tennessee Department of Environment and Conservation (TDEC) will be the responsibility of DOE.

\subsection{ENERGY SYSTEMS}

Energy Systems, as the facility manager, is responsible for managing and operating ORNL under prime contract to DOE. The WAG 2 RI Project will be monitored and operated by the Environmental Sciences Division (ESD) for the ORNL Environmental Restoration (ER) Program. WAG 2 personnel may consist of Energy Systems employees and subcontractors. Specific roles of WAG 2 personnel responsible for management of waste follow.

\subsubsection{Project Manager}

The project manager is responsible for ensuring implementation of the PWMP and revising the plan if necessary. The project manager is also responsible for contacting the site Waste Management Organization before generating waste and distributing the approved waste management plan to all involved organizations.

The project manager (or designee)

1. contacts the ORNL Waste Management Organization to determine the best approach for transfer and subsequent storage, disposal, and/or treatment options;

2. contacts the plant compliance office for guidance in managing all waste types generated as part of this project;

3. ensures that support staff have taken all proper training to sign and complete waste disposal forms and manage specific waste storage areas; 
4. contacts the ORNL Waste Management Organization to reconfirm the availability of storage and disposal facilities (before the generation of waste).

5. ensures that a waste certification procedure has been prepared for this project, that it has undergone the appropriate review, and is implemented.

The project manager (or designee) distributes the PWMP and/or checklist for review to the ORNL Waste Management Organization, ORNL Environmental Compliance Organization, ORNL ER Program Waste Management Organization, the Central Waste Management Division, Laboratory Certification Official (LCO), Generator Certification Official (GCO) and other staff members or organizations that the project manager deems appropriate.

\subsubsection{Energy Systems Waste Management Organization}

Energy Systems Waste Management Organization (ESWMO) is responsible for providing overall guidance, waste acceptance criteria, policies, and certification requirements for conducting waste management activities and future disposal at the Central Waste Disposal Area. ESWMO coordinates multiplant treatment and storage facilities.

\subsubsection{Energy Systems ORNL Waste Management Organization}

The Waste Management Organization at ORNL is responsible for providing review and approval of the waste management plans. ORNL Waste Management organization is also responsible for picking up the waste material from the waste storage area after characterization of the waste has been performed, and after proper waste disposal forms have been completed by the generator and the GCO. The waste will be transported to an approved storage or treatment facility in accordance with regulatory requirements and Energy Systems policies and procedures.

\subsection{ORNL Environmental Compliance Organization}

ORNL Environmental Compliance Organization provides regulatory guidance towards successfully completing the specific project and reviews and approves the PWMP or checklist.

\subsubsection{Environmental Restoration Central Waste Management Organization}

The Central ER Waste Management Organization is responsible for providing overall guidance and policies for ER waste management activities and reviewing PWMPs and checklists.

\subsubsection{ORNL ER Project Waste Management Manager}

The ORNL ER Project Waste Management Manager (ERPWM) is responsible for implementation of ER waste management policies and procedure for ORNL ER projects. The ER Program WM Manager is also responsible for providing guidance in the preparation of waste management plans, and reviews and approves all PWMP or checklists. 


\subsubsection{Generator Certification Official}

The GCO must be an Energy Systems employee (unless waived by the LCO) who successfully has completed waste generator training for Solid Low Level Waste, Satellite Accumulation Area, and GCO training module [24 to $40 \mathrm{~h}$ Superfund Amendments and Reauthorization Act (SARA)/Occupational Safety and Health Administration (OSHA) and Radiation Worker training I or II is optional.]. An appointment letter from the management of the generator organization must be on file with the Waste Minimization, Planning, and Certification (WMPC) Department for any individual assigned the duties of a GCO, prior to the commencement of these duties.

The GCO

1. serves as the interface between the project, LCO and ORNL Waste Management Organization in addressing generator problems and waste certification requirements;

2. ensures that weekly waste generator reports are completed with a copy sent to the ER WM manager;

3. ensures that monthly tabulated volume of waste generated is completed and mailed to the ER Central Office and a copy sent to the ORNL ER WM Manager;

4. certifies by signing waste disposal forms including: Waste Item Description, Container Packing List, and Waste Pickup Request [The GCO ensures that the waste was packaged in accordance with the waste acceptance criteria.];

5. completes or ensures that a Container Packing List (TX-5749) Form has been completed following the "Container Packing List (TX-5749) Form User Instructions (Appendix C);

6. ensures that generation of wastes is handled and disposed of in accordance with the ORNL waste certification program;

7. provides guidance to generator or packager who processes waste packages rejected by Real Time Radiography (RTR);

8. monitors waste generating and managing activities against an approved certification program by periodic inspections, or audits;

9. makes arrangements with the Radioactive Solid Waste Operations (RSWO) group for the acceptance, pickup, and transfer of the waste package to the disposal/storage site;

10. reviews and approves as specified: nonconformance reports and trends, log-in sheets, training records and facility-specific procedures for the proper packaging and handling of solid low-level waste;

11. ensures that appropriate action is taken within the generator organization to correct any deficiencies noted during reviews or audits of generator waste certification activities;

12. reviews and approves all waste management plans. 


\subsubsection{Laboratory Certification Official}

The individual in the Waste Minimization, Planning, and Certification Department who serves as the point of contact for certification of a given waste stream, reviews and approves PWMPs, checklists, and waste certification procedures. The LCO also conducts inspections, audits, reviews, surveillances, and other assessments of the Solid Low Level Waste (SLLW) certification program.

The LCO for Liquid Waste:

1. Serves as the interface between the waste generator, Waste Management Operations (WMO), and the Office of Environmental Compliance and Documentation (OECD).

2. Develops and issues WAC for all ORNL liquid waste collection and treatment systems. Implements a program to oversee waste generators compliance with WAC.

3. Issues written authorization in answer to special requests with the concurrence of WMO and the Environmental Compliance Section (EC).

4. Conducts liquid waste stream surveys to obtain generation rates, volumes, constituents of waste streams, sources, and applicable RCRA waste codes.

5. Develops and conducts liquid waste certification training for liquid waste generators.

\subsubsection{Generator}

The generator is the responsible Energy Systems employee, subcontractor, or person who physically is generating the waste during remedial investigation or any waste generating activities affiliated with the WAG 2 project.

All persons responsible for packaging or placing waste in containers as part of WAG 2 activities are required to take ORNL Solid Low-Level Waste Generator Training, Hazardous Waste Identification Training and Satellite Accumulation Area Training.

All generators must practice proper waste minimization and comply with this PWMP.

The generator/packager is responsible for:

1. Properly containerizing, packaging, and segregating all waste generated as part of the WAG 2 project in addition to other requirements set forth in other documents;

2. Completes all parts of waste disposal forms (Waste Item Description, Container Packing List, Waste Pickup Request) of which they were responsible for obtaining information from Health Physics;

3. Certifies, by signature, on the Waste Item Description that the waste meets the waste acceptance criteria;

4. Maintaining active communication with the GCO to ensure that any issues in SLLW characterization or certification are promptly brought to the attention of the responsible 
individuals and that any new certification requirements or procedures are promptly instituted in routine operations.

\subsubsection{Radioactive Solid Waste Operations Group (RSWO)}

RSWO is responsible for the following:

1. Provides the planning, organizing, and controlling activities relating to disposal/storage of radioactive solid waste.

2. Supervises the field services connected with the operation of the SWSA disposal/storage facilities.

3. Inspects the waste packages and reviews the generator's documentation for compliance with the Waste Acceptance Criteria (WAC).

4. Approves special packaging, as necessary.

5. Provides transportation of the waste package to the SWSA.

6. Reviews the generator's procedures for compliance to the ORNL on-site transportation manual.

\subsubsection{Hazardous Waste Operation Group (HWOG).}

1. Manages and performs all ORNL hazardous and mixed waste transportation functions and activities.

2. Ensures hazardous and mixed waste activities comply with all applicable EPA and TDEC rules and regulations, DOE orders, and DOT regulations.

3. Reviews Request For Disposal Forms (RFDs) and manifests received from generators or area operators, completes the HWOG portion of the document, and sends document to the Waste Management and Remedial Action Division (WMRAD) Document Management Center.

4. Ensures hazardous or mixed wastes meet the unit's WAC as defined in RCRA permits or WMRAD guidelines.

5. Resolves discrepancies noted on RFDs and manifests.

6. Investigates the origin and composition of wastes as necessary to verify proper characterization.

7. Packages or ensures proper packaging of wastes.

8. Prepares documents and coordinates shipments of hazardous or mixed waste to or from ORNL. 


\section{WASTE GENERATING ACTIVITIES}

Activities that will generate waste are described in the Remedial Investigation Plan for $W A G 2$ (ORNL 1990). Most of the wastes will be generated as the result of

- surface water and groundwater sampling,

- soil and sediment core sampling,

- developing and/or purging monitoring wells,

- biological sampling,

- disposing of PPE,

- disposing of contaminated glassware or sample containers,

- anticontamination covering,

- decontamination of sampling equipment, and

- sample residuals returned from the analytical laboratory (internal or external) following analysis.

WAG 2 wastes that cannot be classified or managed as IDW will be managed under best management practices and existing procedures by the Energy Systems/ORNL organizations. These wastes could include

- laboratory operations waste:

- wash water for glassware, ISCO bottles, etc.,

- sample residues,

- disposable PPE,

- dilute acids,

- dilute bases,

- sharps (needles), and

- autoclaved animal blood.

- decontamination and cleaning facility operations wastes:

- decontamination water,

- dilute acids ( $\mathrm{HCL}, \mathrm{HNO}_{3}$ ),

- dilute bases $(\mathrm{NaOH})$, and

- contaminated wipes and rags.

- field instrument maintenance and standardization wastes:

- expended alkaline batteries,

- expended lead-acid batteries, and

- $\quad$ pH buffers.

\subsection{WASTE SOURCES}

\subsubsection{Soilsediment Core Sampling}

Soil/sediment core sampling results in a volume of sample that is in excess of the volume needed for analysis. The surplus sample then becomes waste material. Other waste such as contaminated polyvinylchloride piping, wood, and disposable PPE are also generated. All 
wastes are then placed in approved containers that will be transported to the radioactive waste collection area for final disposition. The expected volumes of liquid and solid compactible waste are listed in Table 1.

\subsubsection{Developing and/or Purging Monitoring Wells}

Groundwater removed from existing wells and from new monitoring wells may contain from $<1$ to $>25 \%$ solids, depending on well construction parameters and the intercepted strata. The liquid fraction of these wastes may contain radionuclides, but no data are available on other potential hazardous contaminants. It is expected that solids generally will be larger than the respirable range of particulate size and settle rapidly in collection vessels. The expected volume of liquid and solid compactible waste is listed in Table 1.

\subsubsection{Surface Water and Seep Sampling}

The volume of surface water collected during sampling may be in excess of volumes necessary for laboratory analysis. Contaminated tubing, wipes, and disposable PPE are also generated during sampling activities. Expected volumes of liquid and solid compactible wastes are listed in Table 1.

\subsubsection{Biological Sampling}

Carcasses of small mammals and birds, which will not be used for analysis, shall be stored until further disposal information from the ESD Environmental Protection Officer (EPO). Processing of biota may result in waste needles that will be disposed of in an approved sharps container. Waste blood from trapped animals will be autoclaved and disposed of as autoclaved biological waste in accordance with guidance from the ORNL Industrial Waste Program Coordinator and EPM-6.1. Animal carcasses, which have originated in radiological areas, will be monitored for radioactivity (gamma)

prior to disposal. The expected volumes of biological wastes have not been predicted due to the nominal amounts potentially produced.

\subsubsection{Disposing of PPE}

Field personnel wear PPE to prevent potentially radioactive materials from contacting the skin and entering the body. The basic set of PPE (Level 1) required in the field includes DOE khakis or washable cotton coveralls that shall be sent to laundry to be cleaned. If field clothing has become contaminated, the clothing must be placed in a yellow and magenta radioactive waste bag for Health Physics survey and tagging. Laundry then picks up contaminated clothing. Disposable gloves are disposed of as solid low-level compactible waste.

\subsubsection{Decontaminating Small Tools By Hand Techniques}

Sampling tools are subjected to initial gross decontamination at the sampling site before transport to a central decontamination or cleaning facility in the laboratory. Small volumes of deionized water and hand wipes are used to remove gross contamination in the field. Decontamination water may contain radiological contaminants, distilled water, or solvents. If waste contains methanol, the mixture shall contain levels $<10 \%$ by volume and will not demonstrate the characteristic of ignitibility. These wastes shall be treated as nonhazardous 
waste or low level radiological waste. The decontamination water shall be contained in an approved carboy or container and the contaminated wipes shall be placed in a radioactive waste bag. Carboys will be stored in the laboratory's radioactive waste area. Expected volumes of liquid and solid compactible waste are listed in Table 1 by task.

\subsubsection{Sample Residual from Analytical Laboratory}

Sample residuals (e.g., soils, sediments, water) that are not consumed by the analytical laboratory (external or internal) will be transferred to Energy Systems Waste Operations for disposal when no longer needed or following archival. 
Table 1. Estimated types and volume of waste

\begin{tabular}{|c|c|c|c|c|c|}
\hline Task & Waste stream/source & Type & Category ${ }^{n}$ & $\begin{array}{l}\text { Volume } \\
\text { (annual) }\end{array}$ & Suspected contaminants \\
\hline \multirow{4}{*}{$\begin{array}{l}\text { Seeps and } \\
\text { tributanes } \\
\text { (water sampling) }\end{array}$} & Residual samples & Liquid & Radıological & $87 \mathrm{ft}^{3}$ & $\begin{array}{l}\text { H-3, Sr-90, Co-60, Cs- } \\
137, \text { U-238/234/232, } \\
\text { Cm-243 }\end{array}$ \\
\hline & Laboratory analysis & Liquid & $\begin{array}{l}\text { Radiological } \\
\text { nonhazardous }\end{array}$ & $05 \mathrm{ft}^{3}$ & $\begin{array}{l}\mathrm{H}-3, \mathrm{Sr}-90, \mathrm{Co}-60, \mathrm{Cs}- \\
137, \mathrm{U}-238 / 234 / 232 \\
\mathrm{Cm}-243, \mathrm{KMnO}_{4}\end{array}$ \\
\hline & & & Radiological & $11 \mathrm{ft}^{3}$ & $\mathrm{H}-3$ \\
\hline & $\begin{array}{l}\text { Decontamination, } \\
\text { personal protective } \\
\text { equipment (PPE), } \\
\text { sample containers }\end{array}$ & $\begin{array}{l}\text { Plastıc, paper, } \\
\text { glass }\end{array}$ & Radiological & $197 \mathrm{ft}^{3}$ & $\begin{array}{l}\text { Sr-90, Co-60, Cs-137, } \\
\text { U-238/234/232, } \\
\text { Cm-243 }\end{array}$ \\
\hline \multirow{7}{*}{$\begin{array}{l}\text { Hydrologıc } \\
\text { pathways } \\
\text { (groundwater } \\
\text { sampling) }\end{array}$} & $\begin{array}{l}\text { Well dniling } \\
\text { decontamination }\end{array}$ & Liquid & Radıological & $67 \mathrm{ft}^{3}$ & $\begin{array}{l}\mathrm{H}-3, \mathrm{Sr}-90, \mathrm{Co}-60, \mathrm{Cs}- \\
137\end{array}$ \\
\hline & Drilhng & Sludge/soll & Radiological & $29 \mathrm{ft}^{3}$ & Sr-90, Co-60, Cs-137 \\
\hline & $\begin{array}{l}\text { Decontamination, } \\
\text { PPE }\end{array}$ & $\begin{array}{l}\text { Plastic, paper, } \\
\text { glass }\end{array}$ & Radıological & $10 \mathrm{ft}^{3}$ & $\begin{array}{l}\text { Sr-90, Co-60, } \\
\text { Cs-137 }\end{array}$ \\
\hline & $\begin{array}{l}\text { Water sampling } \\
\text { residual samples }\end{array}$ & Liquid & Radıological & $0.35 \mathrm{ft}^{3}$ & $\begin{array}{l}\mathrm{H}-3, \mathrm{Sr}-90, \mathrm{Co}-60, \mathrm{Cs}- \\
137\end{array}$ \\
\hline & Laboratory analysis & & $\begin{array}{l}\text { Radiological } \\
\text { nonhazardous }\end{array}$ & $035 \mathrm{ft}^{3}$ & $\begin{array}{l}\mathrm{H}-3, \mathrm{Sr}-90, \mathrm{Co}-60, \mathrm{Cs}- \\
137, \mathrm{KMnO}_{4}\end{array}$ \\
\hline & & & Radıologıcal & $07 \mathrm{ft}^{3}$ & $\mathrm{H}-3$ \\
\hline & $\begin{array}{l}\text { Decontamination, } \\
\text { PPE }\end{array}$ & $\begin{array}{l}\text { Plastic, paper, } \\
\text { glass }\end{array}$ & Radiological & $6 \mathrm{ft}^{3}$ & $\begin{array}{l}\text { Sr-90, Co-60, } \\
\text { Cs }-137\end{array}$ \\
\hline \multirow{3}{*}{$\begin{array}{l}\text { Sedıment } \\
\text { transport } \\
\text { (storm water } \\
\text { sampling) }\end{array}$} & $\begin{array}{l}\text { Water sampling } \\
\text { resıdual samples }\end{array}$ & $\begin{array}{l}\text { Liquid (storm } \\
\text { water) }\end{array}$ & Radiological & $551 \mathrm{ft}^{3}$ & $\begin{array}{l}\text { H-3, Sr-90, Co-60, Cs- } \\
137\end{array}$ \\
\hline & Decontammation & Lqquid & Radiological & $288 \mathrm{ft}^{3}$ & $\begin{array}{l}\mathrm{H}-3, \text { Sr-90, Co-60, Cs- } \\
137\end{array}$ \\
\hline & $\begin{array}{l}\text { Decontammation, } \\
\text { PPE }\end{array}$ & $\begin{array}{l}\text { Plastıc, paper, } \\
\text { glass, } \\
\text { aluminum }\end{array}$ & Radıological & $289 \mathrm{ft}^{3}$ & Cs- 137, Co-60, Sr-90 \\
\hline $\begin{array}{l}\text { Hydrauhc head } \\
\text { monitoring } \\
\text { stations }\end{array}$ & $\begin{array}{l}\text { Well purging and } \\
\text { water sampling }\end{array}$ & $\begin{array}{l}\text { Liquid } \\
\text { (possible pH } \\
>12 \text { ) }\end{array}$ & Radiological & $861 \mathrm{ft}^{3}$ & $\begin{array}{l}\mathrm{H}-3, \mathrm{Sr}-90, \mathrm{Co}-60, \mathrm{Cs}- \\
137\end{array}$ \\
\hline \multirow[t]{2}{*}{$\begin{array}{l}\text { Floodplain } \\
\text { soll/sediment }\end{array}$} & $\begin{array}{l}\text { Soll and sediment } \\
\text { core residual samples }\end{array}$ & $\begin{array}{l}\text { Soll and } \\
\text { sediment }\end{array}$ & Radıological & $2 \mathrm{ft}^{3}$ & $\begin{array}{l}\text { Sr-90, Co-60, } \\
\text { Cs-137, Pu-239/240 }\end{array}$ \\
\hline & $\begin{array}{l}\text { Decontamination, } \\
\text { PPE }\end{array}$ & $\begin{array}{l}\text { Plastic, paper, } \\
\text { PVC piping, } \\
\text { glass and } \\
\text { wood }\end{array}$ & Radıological & $9.8 \mathrm{ft}^{3}$ & $\begin{array}{l}\text { Sr-90, Co-60, } \\
\text { Cs-137, Pu-239/240 }\end{array}$ \\
\hline
\end{tabular}


Table 1. (continued)

\begin{tabular}{|c|c|c|c|c|c|}
\hline Task & Waste stream/source & Type & Category & $\begin{array}{l}\text { Volume } \\
\text { (annual) }\end{array}$ & Suspectod contaminants \\
\hline $\begin{array}{l}\text { Floodplain } \\
\text { soil/sediment } \\
\text { (cont.) }\end{array}$ & Decontamination & Liquid & Radıologıcal & $23.2 \mathrm{ft}^{3}$ & $\begin{array}{l}\text { Sr-90, Co-60, } \\
\text { Cs-137, Pu-239/240 }\end{array}$ \\
\hline \multirow{4}{*}{$\begin{array}{l}\text { Trubutary } \\
\text { assessment } \\
\text { task } \\
\text { (water sampling) }\end{array}$} & Residual samples & Liquid & Radıologıcal & $244 \mathrm{ft}^{3}$ & $\begin{array}{l}\text { H-3, Sr-90, Co-60, Cs- } \\
137, \mathrm{U}-238 / 234 / 232\end{array}$ \\
\hline & Laboratory analysis & & $\begin{array}{l}\text { Radiological } \\
\text { nonhazardous }\end{array}$ & $06 \mathrm{ft}^{3}$ & $\begin{array}{l}\mathrm{H}-3, \mathrm{Sr}-90, \mathrm{Co}-60, \mathrm{Cs}- \\
137, \mathrm{U}-238 / 234 / 232 \\
\mathrm{KMnO}_{4}\end{array}$ \\
\hline & & & Radıological & $1.2 \mathrm{ft}^{3}$ & H-3 \\
\hline & $\begin{array}{l}\text { Decontamination, } \\
\text { PPE, sample } \\
\text { containers }\end{array}$ & $\begin{array}{l}\text { Plastıc, paper, } \\
\text { glass }\end{array}$ & Radıological & $355 \mathrm{ft}^{3}$ & $\begin{array}{l}\text { Sr-90, Co-60, } \\
\text { Cs-137, U-238/234/232 }\end{array}$ \\
\hline \multirow[t]{2}{*}{ Groundwater } & $\begin{array}{l}\text { Redevelopment of } \\
\text { wells }\end{array}$ & Liquid & Radıologıcal & $1003 \mathrm{ft}^{3}$ & $\begin{array}{l}\mathrm{H}-3, \mathrm{Sr}-90, \mathrm{Co}-60, \mathrm{Cs}- \\
137\end{array}$ \\
\hline & & $\begin{array}{l}\text { Plastic, paper, } \\
\text { glass }\end{array}$ & Radıological & $60 \mathrm{ft}^{3}$ & Sr-90, Co-60, Cs-137 \\
\hline
\end{tabular}

${ }^{-}$Hazardous, mxed, nonhazardous, radiological, transuranic, Toxic Substances Control Act, etc 


\section{TYPES AND VOLUME OF WASTE}

Table 1 summarizes the waste types and quantities to be generated by each task within the WAG 2 and site inspection project in approximately a 1-year period. This information is an estimate based on current sampling frequency and practices. As applicable, the wastes are categorized as hazardous, radioactive, nonhazardous, or mixed wastes and information concerning their physical states (such as liquid, solid, or compactible) is included.

Tables 2 and 3 include a radiological characterization of the liquid and solid (soil/sediment) waste where data were available. As data becomes available it shall be incorporated into this plan. Toxicity Characteristic Leaching Procedure (TCLP) tests performed on soil and sediment samples collected on the WOC floodplain, WOL, WOC Weir and Melton Branch Weir indicate that hazardous material levels are well below guidelines for designation as Hazardous Waste (Table 4). 
Table 2. Radiological characterization of liquid waste (Radiological concentrations of liquid waste will be based on a mixture of residual environmental samples and diluted decontamination water. This table includes data from environmental water samples that will be incorporated into the waste stream.)

\begin{tabular}{|c|c|c|c|c|}
\hline Radionuclide & \# of Samples & $\operatorname{Mean}^{d}(\mathrm{pCi} / \mathrm{L})$ & Minimum $^{e}(\mathrm{pCi} / \mathrm{L})$ & Maximum $(p C i / L)$ \\
\hline H-3 & 253 & 4420900 & 0 & 126696000 \\
\hline Sr-90 & 253 & 11199.73 & 0 & 1181395 \\
\hline Co-60 & $\begin{array}{l}112 \text { (dissolved) }^{b} \\
109 \text { (particulate) }^{c}\end{array}$ & $\begin{array}{l}94.7 \text { (dissolved) } \\
6.1 \text { (particulate) }\end{array}$ & 0 & $\begin{array}{l}1880 \text { (dissolved) } \\
208 \text { (particulate) }\end{array}$ \\
\hline Cs-137 & $\begin{array}{l}112 \text { (dissolved)b } \\
109 \text { (particulate) }^{c}\end{array}$ & $\begin{array}{l}11.6 \text { (dissolved) } \\
10.4 \text { (particulate) }\end{array}$ & $\begin{array}{l}<0.7 \text { (dissolved) } \\
0.1 \text { (particulate) }\end{array}$ & $\begin{array}{l}216 \text { (dissolved) } \\
305.5 \text { (particulate) }\end{array}$ \\
\hline Gross alpha & 287 & 1258.3 & $\mathbf{0}$ & 39350 \\
\hline Gross beta & 287 & 55652.41 & 0 & 2816200 \\
\hline U-238 & 16 & 26.27 & 0.02 & 101 \\
\hline U-235 & 16 & 2.41 & $\mathbf{0}$ & 15 \\
\hline U-234 & 16 & 523.03 & 0.03 & 4140 \\
\hline U-232 & 7 & 60.46 & $\mathbf{0}$ & 225 \\
\hline Th-232 & 16 & 0.64 & 0 & 9.9 \\
\hline Th-230 & 16 & 0.90 & 0.02 & 12.7 \\
\hline Th-228 & 16 & 1.74 & 0 & 9.9 \\
\hline Pu-239 & 16 & 2.36 & 0 & 36.7 \\
\hline Pu-238 & 16 & 2.07 & 0 & 31.1 \\
\hline Cm-243 & 17 & 8.50 & 0 & 56.57 \\
\hline Am-241 & 17 & 3.02 & 0 & 22.04 \\
\hline
\end{tabular}

\# of samples = number of sample results used in summary.

${ }^{b}$ Dissolved = Cobalt and Cesium concentrations for water samples that have been filtered.

${ }^{c}$ Particulate $=$ Cobalt and Cesium concentrations in suspended particulates contained in environmental water samples.

d Mean= average concentrations of radionuclides present in residual environmental water samples.

- Minimum = minimum radiological concentration found in environmental water samples added to waste stream.

$f$ Maximum $=$ maximum radiological concentration found in environmental water samples added to waste stream. 
Table 3. Radiological characterization of solid waste (soil/sediment) (Radiological concentrations found in soil/sediment included in solid low-level compactible waste.)

\begin{tabular}{lllll}
\hline \hline Radionuclide & \# of Samples & Mean $^{\mathrm{b}} \mathrm{pCi} / \mathrm{g}$ & $\begin{array}{l}\text { Minimum }^{\mathrm{c}} \\
\text { pCi/g }\end{array}$ & $\begin{array}{l}\text { Maximum }^{\mathrm{d}} \\
\mathrm{pCi}^{\mathrm{g}} \mathrm{g}\end{array}$ \\
\hline \hline Co-60 & 334 & 36.17 & 0.47 & 431.58 \\
Cs-137 & 334 & 1163.31 & $<1.1$ & 25230.09 \\
U-233/234 & 23 & 2.66 & 0.27 & 8.50 \\
U-235 & 23 & 0.07 & 0.02 & 0.20 \\
U-238 & 23 & 1.14 & 0.16 & 1.90 \\
Total Radio-Sr & 23 & 27.16 & 0.07 & 190.00 \\
Th-228 & 23 & 2.09 & 0.38 & 6.50 \\
Th-230 & 23 & 0.89 & 0.47 & 1.30 \\
Th-232 & 1.03 & 0.43 & 1.60 \\
Pu-238 & 23 & 0.43 & 0.01 & 1.60 \\
Pu-239/240 & 23 & 6.07 & 0.02 & 71.00 \\
Am-241 & 23 & 1.84 & 0.11 & 6.16 \\
Cm-244 & 23 & 0.60 & 0.02 & 9.80 \\
\hline \hline
\end{tabular}

a \# samples = number of sample results used in summary.

${ }^{b}$ Mean = average concentrations of radionuclides present in residual environmental soil/sediment samples.

${ }^{c}$ Min $=$ Minimum radiological concentrations found in environmental soil/sediment samples.

${ }^{d} \mathrm{Max}=$ Maximum radiological concentration found in environmental soil/sediment samples. 
Table 4. Average results of Toxicity Characteristic Leaching Procedure tests performed on soil and sediment samples collected on the White Oak Creek floodplain,

White Oak Lake, WOC Weir and Melton Branch Weir

\begin{tabular}{|c|c|c|}
\hline Analyte & $\begin{array}{l}\text { Maximum concentration } \\
(\mathrm{mg} / \mathrm{kg})\end{array}$ & $\begin{array}{l}\text { Regulatory } \\
\text { Level } \\
\text { (mg/kg) }\end{array}$ \\
\hline Benzene & $<0.025$ & 0.500 \\
\hline Carbon tetrachloride & $<0.025$ & 0.500 \\
\hline Chlorobenzene & $<0.025$ & 100.0 \\
\hline Chloroform & $<0.025$ & 6.000 \\
\hline 1,2-Dichloroethane & $<0.025$ & 0.500 \\
\hline 1,1-Dichloroethene & $<0.025$ & 0.700 \\
\hline Methyl Ethyl Ketone & $<0.500$ & 200.0 \\
\hline Tetrachloroethene & $<0.025$ & 0.700 \\
\hline Trichloroethene & $<0.025$ & 0.500 \\
\hline Vinyl Chloride & $<0.050$ & 0.200 \\
\hline 1,4- Dichlorobenzene & $<0.008$ & 7.500 \\
\hline 2-Methylphenol & $<0.008$ & \\
\hline 3-Methylphenol & $<0.008$ & \\
\hline 4-Methylphenol & $<0.008$ & \\
\hline Total-Methyl phenol & $<0.008$ & \\
\hline Hexachloroethane & $<0.008$ & 3.000 \\
\hline
\end{tabular}


Table 4. (continued)

\begin{tabular}{|c|c|c|}
\hline Analyte & $\begin{array}{l}\text { Maximum concentration } \\
(\mathrm{mg} / \mathrm{kg})\end{array}$ & $\begin{array}{l}\text { Regulatory } \\
\text { Level (mg/kg) }\end{array}$ \\
\hline Nitrobenzene & $<0.008$ & 2.000 \\
\hline Hexachlorobutadiene & $<0.008$ & 0.500 \\
\hline 2,4,6-Trichlorophenol & $<0.008$ & 2.000 \\
\hline 2,4,5-Trichlorophenol & $<0.008$ & 400.00 \\
\hline 2,4-Dinitrotoluene & $<0.008$ & 0.130 \\
\hline Hexachlorobenzene & $<0.008$ & 0.130 \\
\hline Pentachlorophenol & $<0.042$ & 100.0 \\
\hline Pyridene & $<0.008$ & 5.000 \\
\hline Gamma-BHC & $<0.00005$ & \\
\hline Heptachlor & $<0.00005$ & 0.008 \\
\hline Endrin & $<0.00010$ & 0.020 \\
\hline Methoxychlor & $<0.00005$ & 10.00 \\
\hline Chlordane & $<0.00010$ & 0.030 \\
\hline Toxaphene & $<0.00500$ & 0.500 \\
\hline Aroclor 1016 & $<0.00208$ & \\
\hline Aroclor 1221 & $<0.00333$ & \\
\hline Aroclor 1232 & $<0.00208$ & \\
\hline Aroclor 1242 & $<0.00208$ & \\
\hline
\end{tabular}


Table 4. (continued)

\begin{tabular}{|c|c|c|}
\hline Analyte & $\begin{array}{l}\text { Maximum concentration } \\
(\mathrm{mg} / \mathrm{kg})\end{array}$ & $\begin{array}{l}\text { Regulatory } \\
\text { Level (mg/kg) }\end{array}$ \\
\hline Aroclor 1248 & $<0.00208$ & \\
\hline Aroclor 1254 & $<0.00208$ & \\
\hline Aroclor 1260 & $<0.00208$ & \\
\hline 2,4-D & $<0.00200$ & 10.0 \\
\hline 2,4,5-TP(Silvex) & $<0.00040$ & 1.00 \\
\hline Arsenic & 0.23 & 5.000 \\
\hline Barium & 1.2 & 100.0 \\
\hline Cadmium & $<0.002$ & 1.000 \\
\hline Chromium & 0.017 & 5.000 \\
\hline Lead & $<0.0160$ & 5.000 \\
\hline Mercury & $<0.0003$ & 0.200 \\
\hline Selenium & 0.032 & 1.000 \\
\hline Silver & $<0.0030$ & 5.000 \\
\hline
\end{tabular}




\section{PROCEDURES FOR MANAGING WASTE}

\subsection{GENERATING}

DOE and Energy Systems applicable control programs governing waste disposal activities will be implemented for this project. These programs include sampling and analysis, waste certification, waste minimization, hazardous waste and radiation worker training, QA, ES\&H, health physics, and configuration management. These programs will ensure compliance with all applicable state, federal, and DOE regulatory requirements including RCRA, CERCLA, SARA, OSHA, and the Department of Transportation (DOT) for the proper handling and control of radioactive and hazardous wastes.

\subsection{SEGREGATING}

All generated waste from field and laboratory activities shall be segregated by each waste type as specified in Table 1. Waste will not be accepted by Waste Operations unless it is properly segregated, packaged, characterized and appropriate documentation is completed. Waste shall be segregated according to ORNL waste management policies and the Waste Acceptance Criteria (ORNL 1994). Waste can be classified according to the following types:

1. Hazardous waste (satellite accumulation area or 90 day area)

A. RCRA Hazardous Waste:

1. Listed as a $\mathrm{K}, \mathrm{P}, \mathrm{U}$, or $\mathrm{F}$ listed waste

2. Exhibits one of the following characteristics

a. an ignitable waste is one with a flash point of $140^{\circ} \mathrm{F}$ or lower

b. a corrosive waste is one that either has a $\mathrm{pH}$ less than or equal to 2 or greater than or equal to 12.5

c. a reactive waste is one that is explosive, gives off toxic gases, or can violently react with water

d. a TCLP toxic waste is one that has the potential to leach certain metals, pesticides, or organics

3. Is not excluded

4. A mixture involving a hazardous waste

B. Mixed waste-hazardous waste and radioactive waste constituents

C. All aerosol cans 
2. Nonhazardous waste

Any waste material that is not RCRA regulated but does not meet disposal standards of harmless to the public and the environment.

3. Radioactive waste

A. Solid radioactive waste: any dry solid material or combination of dry materials contaminated or suspected of being contaminated with radioactive material

1. Compactible low-level waste: paper, plastic, cloth, rubber, small wood and lightweight metal items and glass containers up to 1 gal size.

2. Noncompactible low-level waste: concrete, glass ( $>1$ gal), metal, equipment components, tools, and other large items

B. Liquid radioactive waste: waste containing radioactive constituents exceeding the Derived Concentration Guide (DCG) values that are provided in DOE Order 5400.5 (Table 5)

C. Very low activity waste (VLA): waste that has no measurable external contamination and no specific knowledge of significant quantities of internally contained radioactivity. However, because of its origin or process knowledge cannot be certified as free of radioactivity and may have some identified potential for internal, undetectable radioactive contamination above free release limits. VLA waste must be handled as a category of radioactive waste and stored in a controlled area.

4. $\operatorname{PCB}(\mathrm{s})$ waste

Polychlorinated biphenyl(s); PCBs and PCB items that are subject to the disposal requirements of subpart $D$ of 40 CFR 761.

5. Biological waste

Waste of biological nature (e.g., animal carcass, autoclaved animal blood).

6. Sanitary waste

Waste that contains nonhazardous and nonradioactive constituents to include no chemical wastes (e.g., paper, gloves)

7. Sharps

Examples include needles and razor blades. Needles must be autoclaved and packaged in a puncture-proof container which should be kept in the waste collection area.

Container are then discarded in locked medical/biological waste dumpster.

Any wastes generated in the field or laboratory which may be suspected of having radiological constituents or has originated in a radiologically contaminated area should be treated as Very Low Activity Waste. This waste may have undectable radiological levels present. 


\subsection{LABELING}

All waste containers must be labeled properly in the field and in the laboratory for contamination control, storage, and disposal. A label must be legible and visible on the primary container.

Table 5. Maximum allowable concentration of specific waste water contaminants for discharge to the Process Waste Treatment Plant

\begin{tabular}{|c|c|}
\hline Contaminant & Concentration \\
\hline \multicolumn{2}{|l|}{ Radionuclides } \\
\hline Gross Alpha & $175 \mathrm{~Bq} / \mathrm{L} \quad(4,725 \mathrm{pCi} / \mathrm{L})$ \\
\hline Gross Beta & $10,000 \mathrm{~Bq} / \mathrm{L} \quad(270,000 \mathrm{pCi} / \mathrm{L})$ \\
\hline Sr-90 & $10,000 \mathrm{~Bq} / \mathrm{L}(270,000 \mathrm{pCi} / \mathrm{L})$ \\
\hline Cs-137 & $400 \mathrm{~Bq} / \mathrm{L} \quad(10,800 \mathrm{pCi} / \mathrm{L})$ \\
\hline $\mathrm{Co}-60$ & $185 \mathrm{~Bq} / \mathrm{L}^{a} \quad(4,995 \mathrm{pCi} / \mathrm{L})$ \\
\hline Eu-152 & $740 \mathrm{~Bq} / \mathrm{L}^{a} \quad(19,980 \mathrm{Pci} / \mathrm{L})$ \\
\hline Eu-154 & $740 \mathrm{~Bq} / \mathrm{L}^{a} \quad(19,980 \mathrm{pCi} / \mathrm{L})$ \\
\hline Eu-155 & $3,700 \mathrm{~Bq} / \mathrm{L}^{\mathrm{a}} \quad(99,900 \mathrm{pCi} / \mathrm{L})$ \\
\hline $\mathrm{Ru}-106$ & $222 \mathrm{~Bq} / \mathrm{L}^{a} \quad(5,994 \mathrm{pCi} / \mathrm{L})$ \\
\hline $\mathrm{ZrNb}-95$ & $1,480 \mathrm{~Bq} / \mathrm{L}^{a} \quad(39,960 \mathrm{pCi} / \mathrm{L})$ \\
\hline \multicolumn{2}{|l|}{ Metals } \\
\hline $\mathrm{Ag}$ & $0.4 \mathrm{mg} / \mathrm{L}$ \\
\hline As & $40.0 \mathrm{mg} / \mathrm{L}$ \\
\hline B & $40.0 \mathrm{mg} / \mathrm{L}$ \\
\hline $\mathrm{Ba}$ & $120.0 \mathrm{mg} / \mathrm{L}$ \\
\hline $\mathrm{Be}$ & $0.2 \mathrm{mg} / \mathrm{L}$ \\
\hline $\mathrm{Cd}$ & $0.3 \mathrm{mg} / \mathrm{L}$ \\
\hline $\mathrm{Cr}$ & $7.5 \mathrm{mg} / \mathrm{L}$ \\
\hline $\mathrm{Cu}$ & $2.5 \mathrm{mg} / \mathrm{L}$ \\
\hline $\mathrm{Fe}$ & $500.0 \mathrm{mg} / \mathrm{L}$ \\
\hline $\mathrm{Hg}$ & $0.004 \mathrm{mg} / \mathrm{L}$ \\
\hline $\mathrm{Ni}$ & $65.0 \mathrm{mg} / \mathrm{L}$ \\
\hline $\mathrm{Pb}$ & $30.0 \mathrm{mg} / \mathrm{L}$ \\
\hline $\mathrm{Sb}$ & $65.0 \mathrm{mg} / \mathrm{L}$ \\
\hline $\mathrm{Se}$ & $15.0 \mathrm{mg} / \mathrm{L}$ \\
\hline $\mathrm{Zn}$ & $60.0 \mathrm{mg} / \mathrm{L}$ \\
\hline
\end{tabular}


Table 5. (continued)

\begin{tabular}{lc}
\hline \hline Others & \\
\hline \hline Chlorine $(\mathrm{Cl})$ & $20.0 \mathrm{ppm}$ \\
Cyanide $(\mathrm{CN})$ & $0.2 \mathrm{ppm}$ \\
Nitrate $\left(\mathrm{NO}_{3}\right)$ & $10.0 \mathrm{ppm}$ \\
& \\
Oil and grease & $100.0 \mathrm{ppm}$ \\
$\mathrm{pH}$ & $>6.0$ \\
Phosphate $\left(\mathrm{PO}_{4}\right)$ & $5.0 \mathrm{ppm}$ \\
Sulfate $\left(\mathrm{SO}_{4}\right)$ & $3000.0 \mathrm{ppm}$ \\
Total organic carbon & $50.0 \mathrm{ppm}$ \\
Total suspended solids & $1000.0 \mathrm{ppm}$ \\
Total toxic organics & $100.0 \mathrm{ppm}$ \\
\hline \hline
\end{tabular}

a DOE Order 5400.5 DCG values.

Each label must contain the following:

1. one of the following descriptions:

- hazardous waste

- hazardous waste, mixed waste

- nonhazardous waste

- radioactive waste

2. printed names of the chemical constituents (formulas optional)

- concentration of chemical constituents should be included on the label (if known or best estimate).

3. start data if waste in 90 day area.

\subsection{WASTE PACKAGING, HANDLING, AND DISPOSAL}

Waste containers shall be stored in the designated waste collection area (radioactive collection area, satellite accumulation area, or 90 day area) within each laboratory or field site. Waste must be in approved containers, properly labeled, and appropriate disposal forms filed for waste acceptance by Energy Systems ORNL Waste Management Organization for treatment, storage, or disposal. All waste containers must be placed in secondary containers while in the waste collection areas to prevent spills. This secondary containment shall hold $10 \%$ of the total volume of the liquid waste stored in the area or the volume of the largest container (whichever is larger). 
ESD personnel involved in storage and transfer of waste:

1. ESD Environmental Protection Officer: Marilyn S. Hendricks 4-7296

2. ESD Radiation Protection Officer: Monty Ross 4-7837

3. ESD Generator Certification Officer: Marilyn S. Hendricks 4-7296

4. ESD Health Physics Technician: George Houser 6-1408

5. ESS Generator Certification Officer: Ramie Wilkerson 4-7260

6. Environmental Biotechnology Section Generator Certification Officer: $\quad$ Tom Stephens $\quad 4-0226$

7. EASS Generator Certification Officers: Brian Spalding 4-7265 John Switek $\quad 4-7415$

\subsubsection{Hazardous Or Nonhazardous Waste}

Waste generators shall adhere to the following steps for waste disposal:

1. monitor the amount of waste accumulated (It can take 8-12 weeks for Hazardous Waste Operations Group to pick up waste, so estimate additions to waste accordingly while waiting for pick-up so limits are not exceeded.);

2. contact ESD EPO to obtain necessary forms for disposal of waste;

3. complete Waste Item Description Form (TX-5745)-follow "Waste Item Description (TX5745) Form User Instructions" (Appendix C);

4. complete Waste Pickup Request Form (TX-5753)-follow "Waste Pickup Request (TX-5753) Form User Instructions" (Appendix C);

5. complete Container Packing List Form (TX-5749), if required-follow "Container Packing List (TX-5749) Form User Instructions" (Appendix C);

6. contact the Health Physics Technician to tag the waste for disposal and sign the Waste Item Description Form and Container Packing List;

7. photocopy completed forms to be kept in log book;

8. submit the Waste Pickup Request, Container Packing List and corresponding Waste Item Description Forms to the ESD EPO, who will review and submit forms to waste management; and

9. deposit waste in closed primary container that is properly labelled as hazardous or nonhazardous waste. 


\subsubsection{Liquid Radioactive Waste}

Comply with the following steps:

1. Notify LCO (John Parrott) to determine acceptability of waste at ORNL Treatment Facility;

2. call Chris Scott (4-7057) for waste pick up if liquid waste is below the Derived Concentration Guide values given in DOE Order 5400.5;

3. contact the Radiation Officer for disposal forms if liquid waste is equal to or above the Derived Concentration Guide values given in DOE Order 5400.5;

4. complete Waste Item Description Form (TX-5745)-follow "Waste Item Description (TX5745) Form User Instructions";

5. complete Waste Pickup Request Form (TX-5753)-follow "Waste Pickup Request (TX5753) Form User Instructions";

6. complete Container Packing List Form (TX-5749). Follow "Container Packing List (TX5749) Form User Instructions";

7. contact the Health Physics Technician to tag the waste for disposal and complete and sign appropriate sections of the Waste Item Description Form and Container Packing List;

8. photocopy completed forms to be kept in log book; and

9. submit the Waste Pickup Request, Container Packing List, and corresponding Waste Item Description Forms to the ESD EPO.

\subsubsection{Compactible Low-Level Waste}

Comply with the following steps:

1. segregate low-level waste and collect in a liquid-free container;

2. place bagged waste in a clear radioactive waste bag found in Room 144 and seal with radiation warning tape-do not seal until Health Physics has surveyed the waste (Note: Solid Radioactive Waste should be double bagged.);

3. complete Waste Item Description Form (TX-5745)-follow "Waste Item Description (TX5745) Form User Instructions";

4. complete Waste Pickup Request Form (TX-5753)-follow "Waste Pickup Request (TX5753) Form User Instructions"

5. complete Container Packing List Form (TX-5749)-follow "Container Packing List (TX5749) Form User Instructions"; 
6. contact the Health Physics Technician to tag the waste for disposal and complete and sign appropriate sections of the Waste Item Description Form and Container Packing List;

7. photocopy completed Forms to be kept in log book; and

8. submit the Waste Pickup Request and corresponding Waste Item Description Forms to the ESD GCO who will ensure forms are properly submitted.

\subsubsection{Noncompactible Low-Level Waste}

Comply with the following:

contact the ESD Generator Certification Officer for disposal information.

\subsubsection{PCB Waste}

Contact the ESD EPO for disposal information. The production and management of PCB waste must be handled case by case because of strict regulations governing PCB research. Reference materials include EPM 3.6 and EPA 40 CFR 761.30(j).

\subsubsection{Biological Waste}

Complete the Waste Item Description Form (TX-5745) and contact ESD EPO for further disposal information.

\subsubsection{Sanitary Waste}

1. Most common items not acceptable for green dumpsters:

- RCRA hazardous and/or mixed waste

- TSCA waste (PCBs)

- radioactive waste

- liquid wastes

- wastes containing free liquids

- classified wastes

- bulk metals (large quantities of metals-e.g., large pipe)

- lead acid, Nickel/Cadmium, and Silver and Lithium batteries

- Mercury-contaminated waste

- tires

- fluorescent light bulbs

- incandescent light bulbs

2. Most common items that are acceptable for green dumpsters

- paper

- wood ( $<6 \mathrm{ft}$ in length)

- plastic and rubber

- organic garbage (food, leaves, etc.)

- textile products 
- incidental metals (small amounts of metals-e.g., metal bottle cap)

- HVAC air filters

- alkaline batteries

- button-type batteries

White paper and cardboard are recyclable and should not be disposed of in green dumpsters.

3. Glass dumpsters

- glass [triple rinsed - only P listed or K, P, U, or F listed waste with a (H) Hazard Code containers need to be triple rinsed].

4. Waste requiring special handling and preapproval for disposal

- dead animals

- asbestos waste

- fiberglass with loose fibers

- empty paint cans

- empty pesticide containers

- aerosol cans (should be routed through the WMOG for proper purging of contents and processing for scrap metal.)

- autoclaved/packaged noninfectious medical waste (labelled with generation site, generator's name and phone number; contact Marilyn Hendricks for disposal)

4. For items not appearing on this list, contact the ESD EPO for proper disposal instructions.

\subsection{RADIOACTIVE WASTE CHARACTERIZATION}

Curie content must be determined and documented for each waste package. Following are some acceptable methods of determining curie content as directed by ER Program Waste Management.

Note: The type of instrument used to obtain exposure rates and any calculations performed for this purpose must be documented and retained with the generator's waste disposal records.

\subsubsection{Isotope(s) is Known}

If mixtures of isotopes are present, the ratio of the mixture must also be known to use the following methods:

Process Knowledge - This method is best suited to production operations. Process knowledge must be based on evaluation of the waste stream. Documentation determining curie content must be retained with associated disposal forms.

Materials Accountability - A known amount of radioactive material is introduced into the experiment (or process). A known amount of radioactive material is recovered form the experiment. The amount of radioactive material unaccounted for is assumed to be waste. 
Disintegrations Per Minute to Curie Conversion (best suited to low levels of activity) - A known efficiency must exist (or be established) for the radioisotope and instrument combination to be used. Radiation emissions are measured in counts per minute (cpm) and converted to disintegrations per minute (dpm). If background cpm is a measurable portion of the total cpm, it must be subtracted from the total prior to converting to dpm. Once dpm is determined, curie content can be estimated.

Example: A portable beta-gamma instrument has been obtained that yields approximately $30 \mathrm{dpm} / \mathrm{cpm}$ at contact for ${ }^{238} \mathrm{U}$. A waste package containing only ${ }^{238} \mathrm{U}$ is monitored with the same instrument to emit $1000 \mathrm{cpm}$ beta-gamma above background. How many curies of ${ }^{238} \mathrm{U}$ are estimated to be in the waste?

$$
\begin{aligned}
\mathrm{dpm} & =(1000 \mathrm{cpm}) \times(30 \mathrm{dpm} / \mathrm{cpm}) \\
& =30,000 \mathrm{dpm} \\
\text { curies } & =\mathrm{dpm} / 2.22 \times 10^{12} \\
& =30,000 \mathrm{dpm} / 2.22 \times 10^{12} \\
& =1.35 \times 10^{-8}{ }^{238} \mathrm{U}
\end{aligned}
$$

Exposure to Curie Conversion (best suited to point sources detectable at greater that one foot distances) - In order to estimate the number of curies, the gamma exposure rate from a single radioisotope at a distance of one foot from the source must be known.

\subsubsection{Point Source Relationship}

The distance at which an exposure rate can be measured must be at least five times the longest dimension of the point source. In other words, if the longest dimension of a point source is greater than 2.4 inches ( $1 / 5 \times 12$ inches), the exposure rate must be measured at a distance greater than one foot to remain accurate. Measuring and converting exposure rate from distances greater than one foot to a one foot distance can be done as follows:

$$
R_{2}=\left(d_{1}\right)^{2}\left(R_{1}\right) /\left(d_{2}\right)^{2}
$$

where:

$\mathbf{R}_{2}=$ Unknown exposure rate (rads/hour) at desired distance

$\mathrm{d}_{1}=$ Measured distance (feet) of known exposure rate

$\mathrm{R}_{1}=$ Measure exposure rate (rads/hour) at known distance

$\mathrm{d}_{2}=$ Desired distance (feet) of unknown exposure rate

Example: A point source 4 inches in diameter has a gamma exposure rate of 0.010 rads/hour (10 millirads/hour) at a distance of 20 inches $(5 \times 4$ inches $\times 1 \mathrm{ft} / 12$ inches $=1.67$ feet). What is the exposure rate at a distance of 1 foot?

$$
\begin{aligned}
\mathbf{R}_{2} & =(1.67 \mathrm{ft})^{2}(0.010 \mathrm{rads} / \mathrm{hour}) /(1 \mathrm{ft})^{2} \\
& =0.03 \mathrm{rads} / \text { hour } \\
& =30 \text { millirads/hour @ } 1 \text { foot away from the source }
\end{aligned}
$$




\subsubsection{Determining the Number of Curies}

For point source gamma emitters with energies between 0.07 and $4 \mathrm{MeV}$, the number of curies can be estimated within $\pm 20 \%$. The MeV energies of known radionuclides can be obtained from various text, including Knolls Atomic Chart Of The Nuclides and the David C. Kocher Handbook of Radioactive Decay Data Tables. The following method is currently acceptable to estimate the number of curies contained in gamma contaminated radioactive waste:

$$
\begin{aligned}
& \mathrm{C}=\mathrm{R} / 6 \mathrm{E} \\
& \text { where: } \\
& \mathrm{C}=\text { Number of Curies } \\
& \mathrm{R}=\text { Exposure Rate (rads/hour) @ } 1 \text { foot } \\
& \mathrm{E}={ }^{*} \text { Total MeV Energy of Isotope } \\
& 6 \quad=\text { conversion factor }
\end{aligned}
$$

* Note: for an isotope which is decaying with more than one energy, each MeV energy must be multiplied by its own intensity. The sum of the energies will be used as $\mathrm{E}$ above.

Example: A package of waste containing ${ }^{22} \mathrm{Na}$ has a measured (or calculated) gamma exposure rate of $\mathbf{3 0}$ millirads/hour at a distance of 1 foot from the source. The most abundant gamma decay energy for ${ }^{22} \mathrm{Na}$ is $1.275 \mathrm{MeV}$. How many curies of ${ }^{22} \mathrm{Na}$ are contained in the waste?

$$
\begin{aligned}
\mathrm{C} & =(0.03 \mathrm{rads} / \mathrm{hour}) / 6(1.275 \mathrm{MeV}) \\
& =3.92 \times 10^{-3} \text { curies }
\end{aligned}
$$

\subsubsection{Isotope(s) is Unknown}

Sampling and analysis (radioassay) must be used whenever unknown isotopes or unknown mixtures of isotopes are present as follows:

Either the entire waste package, or an aliquot from a homogeneous waste package must be analyzed to either identify the isotope, or determine the curie content. If the analysis is used only to identify the isotope, one of the methods listed above can be used to determine the number of curies. Documented analytical results must be attached to applicable waste disposal forms. 


\section{WASTE MINIMIZATION}

All efforts shall be taken to minimize generation of all wastes during the course of this project. As a minimum, the following actions will be taken:

1. All generated waste from field and laboratory activities shall be segregated as indicated in Table 1 to minimize the disposal of nonrecyclable noncontaminated, nonhazardous wastes. Wastes will not be accepted by Waste Operations unless it is properly segregated, labeled, characterized, and the appropriate paperwork completed.

2. Presampling planning, Health Physics and Industrial Hygiene contamination control measures, and worker awareness will support minimizing the mixing of waste types.

3. When possible, materials that may be contaminated with sediment will be cleaned to produce noncontaminated materials. The cleaning process will involve washing and rinsing of the materials to remove the radioactive contamination. Effectiveness of the cleaning will be demonstrated by field surveys of the material with the beta/gamma meter to show compliance with HP requirements. The liquid waste resulting from the washing should be containerized, stored, and disposed of as warranted. Wiping equipment with dry paper towels is preferred because it is efficient and minimizes waste generation. Steps will be taken to use as little water as possible in decontamination operations.

4. Field personnel shall wear Level 1 PPE that includes DOE khakis, washable cotton coveralls, disposable gloves, and boots. Activities (i.e., sediment coring) that can expose project personnel to higher levels of contaminants will require additional sets of clothing such as Contamination Zone coveralls (C-Zones) and an additional set of gloves that shall be taped to the C-Zones. Protective equipment will be chosen to minimize the amount of waste material generated and to enable recycling of as much PPE as possible.

5. Non-hazardous chemicals should be used whenever possible. 


\section{ENVIRONMENTAL HEALTH AND SAFETY}

All issues concerning the ES\&H of the WAG 2 RI project are defined in the Health and Safety Plan for the Remedial Investigation of the Waste Area Grouping 2 at the Oak Ridge National Laboratory, Oak Ridge, Tennessee (Cofer et al. 1993).

\subsection{CONTAMINATION CONTROL}

Surveillance by Health Physics or the WAG 2 Site Safety and Health Officer (SSHO) will be conducted during WAG 2 sampling and laboratory activities to identify any conditions that may indicate the presence of radioactive contamination or RCRA hazardous wastes. If warranted, appropriate tests and analysis will be conducted. The GCO as designated, authorized and qualified by ESD Waste Management, will be responsible for determining whether additional testing or sampling is required by Energy Systems. Wastes will be properly handled and segregated into carboys or radioactive material bags to prevent the spread, mixing, or loss of any soil or liquid containing radiologically contaminated material. Waste containers should be secondarily contained and placed over plastic sheeting when appropriate at laboratory or field sites. Contamination control will also be maintained by the utilization of proper PPE and frisking procedures to detect radiological contamination on project personnel and equipment.

\subsection{PERSONNEL EXPOSURE CONTROL}

The Health Physicist or the SSHO (or team leader) will accompany field sampling personnel in radiological areas to monitor and control exposure levels while performing sampling activities. The SSHO will perform periodic surveillances and audits during sampling activities to ensure compliance with health and safety procedures. All standard radiation protection measures defined in the WAG 2 Health and Safety Plan will be followed to ensure personnel safety. The degree of radiation protection needed in the field is dependent on the types of areas and sites that are sampled. For example, personnel sampling in radiation areas, $>3 \mathrm{mRem} / \mathrm{h}$, should decrease time spent at that site and may wear coveralls for extra protection from contamination. All work in contaminated areas or areas subject to radiation exposure shall be properly planned and executed so as to keep personnel exposure ALARA. All WAG 2 field sampling personnel shall be participants in the Baseline Bioassay Program and the Whole Body Monitoring Program to monitor internal exposure of radionuclides.

\subsection{SPILL CONTROL}

Spills will be controlled and cleaned up where and when they occur. The first responder shall perform the following basic steps:

- isolate the area;

- contain the spill (if possible); 
- achieve containment using sorbent boom, diking material, silica absorbent material, or combination of these (other material may be used in an emergency but manufactured absorbent materials are preferred);

- pick up spilled material with appropriate absorbent (spill kits will be kept in a close proximity to the work site);

- decontaminate the area (if deemed necessary by the Health Physicist);

- containerize sorbent or waste material in DOT or disposal site approved container;

- $\quad$ arrange transportation, storage, and disposal with ESD;

- report spills to ORNL Environmental Compliance Division; and

- notify your immediate supervisor or Laboratory Shift Supervisor if a spill cannot be contained or poses any potential risk to personnel.

A spill control kit should contain an adequate amount of the following:

- absorbent pads,

- chemical resistant gloves,

- shovel, and

- broom. 


\section{WASTE COLLECTION AREAS}

All waste collected during WAG 2 field and laboratory activities is stored in the waste collection areas within laboratories or designated field sites. These areas shall be inspected weekly by ESD personnel. The laboratory steward must ensure compliance with regulations and proper procedures for maintenance of these areas.

\subsection{COMPONENTS, LIMITS, AND SETUP OF WASTE COLLECTION AREAS}

The satellite accumulation areas within laboratories shall designated with a sign and outlined with red tape. The maximum volume of waste is limited to a total of 55 gal of hazardous waste, of which 1 quart may be acutely hazardous waste ( $P$ listed waste or a $\mathrm{K}, \mathrm{F}$ or $\mathrm{U}$ listed waste with a $(\mathrm{H})$ Hazard Code.

The nonhazardous waste areas within laboratories shall be designated with a sign and outlined with blue tape. The generator should initiate disposal effort when container approaches capacity.

The radioactive waste area shall be designated with a sign and outlined with yellow tape. The generator should initiate disposal effort when container approaches capacity.

Contact the ESD EPO for instructions on PCB Waste Collection Area setup and instructions on limits.

\subsection{TRAINING}

Training for waste generators will be conducted and documented in accordance with ESD, Laboratory Certification Office, and ER Program policies. Training for all personnel shall include but are not limited to the following:

- Waste Generator Training for Solid Low-Level Waste and

- Waste Generator Training for Satellite Accumulation Area.

- 90 Day Area Training.

Additional training for Waste GCO shall include the following:

- GCO Training Module (only MMES personnel may serve as GCO).

WAG 2 field sampling personnel generating waste shall complete the following:

- $40 \mathrm{~h}$ SARA OSHA Training and

- Radiation Worker training II. 


\section{WASTE TRANSFERRED TO ENERGY SYSTEMS AND FINAL DISPOSITION}

WAG 2 field or designated personnel are responsible for transporting liquid and solid low-level radioactive waste generated in the field to the waste collection areas. Contaminated solid waste will be transferred to Energy Systems Waste Management who will transport it from the waste collection areas to an approved waste disposal, treatment, or storage facility. Liquid waste will be transferred to Energy Systems WMO who will transport it as appropriate to the Nonradiological Wastewater Treatment Plant, Process Waste Treatment Plant, or the Low-Level Liquid Waste system. All transportation activities will be conducted in accordance with applicable MMES or DOT requirements. 


\section{QUALITY ASSURANCE}

QA management considerations have been reviewed and requirements are included in the QA Plan for the WAG 2 project (Atwood and Miller 1992). Energy Systems, in its role as facility manager, will track participant activities and consolidate records, reports, etc., to ensure that project QA activities are performed and required documentation is complete. Surveillances will be conducted as defined in the plan by Energy Systems. All waste disposal forms shall be filled out by the generator and approved and authorized by the project GCO. The GCO shall maintain record copies of all waste disposal forms.

The WAG 2 GCO, in conjunction with ESD, shall also perform and document weekly audits of the field and laboratories maintaining waste collection areas. If a nonconformance is determined, the WAG 2 QA/Quality Control Coordinator shall be notified and formal nonconformance and corrective actions shall be filed with the individual. 


\section{REFERENCES}

ACGIH (American Conference of Governmental Industrial Hygienists) 1991. Threshold Limit Values for Chemical Substances and Physical Agents, Cincinnati, Ohio.

Atwood, G. P. and Miller, D. E., 1992. Quality Assurance Plan for the Remedial Investigation of Waste Area Grouping 2 at Oak Ridge National Laboratory, Oak Ridge, Tennessee. ORNL/ER-134, Oak Ridge, Tenn.

Cofer, G. H., Holt, V. L., and Roupe, G.W. 1993. Health and Safety Plan for the Remedial Investigation of the Waste Area Grouping 2 at Oak Ridge National Laboratory (ORNL), Oak Ridge, Tennessee., Oak Ridge, Tenn.

DOE (U.S. Department of Energy) 1988a. DOE Order 5820.2A, Radioactive Waste Management.

DOE (U.S. Department of Energy) 1988b. DOE Order 5480.11, Radiation Protection for Occupational Workers.

DOE (U.S. Department of Energy) 1990. DOE Order 5400.5, Radiation Protection of the Public and the Environment.

DOE (U.S. Department of Energy) 1992. U.S. Department of Energy Radiological Control Manual, DOE N 5480.6, DOE/EH-0256T.

EPA (U.S. Environmental Protection Agency) 1988. 40 CFR 61, "National Emission Standards for Hazardous Air Pollutants."

EPA (U.S. Environmental Protection Agency) 1990. 40 CFR 300 (55 FR 8756), "National Oil and Hazardous Substances Pollution Contingency Plan: Final Rule."

EPA (U.S. Environmental Protection Agency) 1991. Management of Investigation-Derived Waste During Site Investigation, EPA/540/G-91/009, Office of Research and Development.

Miller, D.E. 1993. Phase I Remedial Investigation Report of Waste Area Grouping 2 at Oak Ridge National Laboratory, Oak Ridge, Tennessee. Draft. DOE/OR/01-1179\&DO.

ORNL (Oak Ridge National Laboratory) 1990. Remedial Investigation Plan for Waste Area Grouping 2 at Oak Ridge National Laboratory, Oak Ridge, Tennessee. ES/ER-14\&D1.

ORNL (Oak Ridge National Laboratory) 1992a. Health Physics Manual: Procedures and Practices for Radiation Protection and Radiation Monitoring, Oak Ridge, Tenn.

ORNL (Oak Ridge National Laboratory) 1992b. Industrial Hygiene Manual: Procedures for ORNL Industrial Hygiene Programs, Industrial Hygiene Section, Oak Ridge, Tenn.

ORNL (Oak Ridge National Laboratory) 1994. Waste Acceptance Criteria for Radioactive Solid Waste Disposal at SWSA-6, WMRA-WMPC-413. Oak Ridge, Tennessee. 
OSHA (Occupational Safety and Health Administration) 1991. 29 CFR 1910.120 "Hazardous Waste Operations and Emergency Response," Waste Management Guidance Document. 

Appendix A 



\section{WASTE MANAGEMENT PLAN CHECKLIST \\ ORNL Environmental Restoration Program}

1. Project Name: Waste Area Grouping 2 Remedial Investigation Project

Responsible Project/WAG Manager: Stephen Herbes

Phone: 574-7336

Address: Building 1505, Rm. 366, MS-6038

Location of Project: Waste Area Grouping 2, surrounding areas, and Laboratories located in Bldg 1505.

Organization/Subcontractor Performing Waste Generation Task: WAG 2 Project personnel (Martin Marietta Energy Systems employees and subcontractors).

Work Order Number for Waste Management/P\&E Activities:

Rm 311: PX048E12

Rm 227: PX050U02

2. Responsible field ORNL Construction Engineer/Prime or Subcontractor:

Not applicable

Responsible field ES\&H Officer: Virginia L. Holt

Phone: 241-5240

Address: Bldg 1506, MS-6351

Map of WAG 2 location may be found in the Project Waste Management Plan.

3. Project Description: Detailed information found in Sections 1.2 and 1.3 within the Project Waste Management Plan.

4. Completion and Signatures of Waste Management Forms:

Project manager is to assure that a minimum of two individuals (laboratory stewards) have had the proper training which will authorize them to sign various waste disposal forms and or manage waste collection areas. All field and laboratory personnel assigned the task of packaging and placing waste in containers are required at a minimum to participate in the ORNL Solid Low Level Waste Generator Training.

Individual signing as "generator" of SLLW shall have been physically present at the time the waste was packaged. 
Individuals who have not taken the proper training authorizing them to sign Waste Disposal forms should contact Fay Frederick at 576-1460 or B. Monty Ross (ESD) at 574-7837, for specific Training Requirements.

A. Waste Item Description Form (TX-5745) (Appendix A, attachment 1):

(Generator)

Marilyn S. Hendricks

(GCO, MMES Employee)

George Houser

(Health Physics Technician)

B. Container Packing List (TX-5749) (Appendix A, attachment 2):

Marilyn S. Hendricks

(Certification Officer: $\mathrm{GCO}$ )

to be assigned

(Waste Management Organization Officer: WMO)

George Houser

(Health Physics Technician)

C. Waste Pickup Request (Appendix A, attachment 3):

Requestor

Packager

5. Person or organization responsible for completing the ER Waste Generation Report:

This report is to be completed weekly and mailed to ORNL ER Waste Management Manager. This report is to be completed by the project manager, and the values are to be reported in cubic feet.

Project Manager: Steve Herbes 
6. Organization or person responsible for providing, MMES, prime or subcontractor with characterization data for waste transfer or dispositioning:

Project Manager: Steve Herbes

7. Organization or person responsible for obtaining samples from waste containers and transporting those samples to the analytical laboratory for characterization purposes:

WAG 2 Analytical Services Coordinator

8. Describe sampling plan for taking samples (from waste containers or the area of contamination) that are to be sent to the lab for characterization information which will be used in completing the waste disposal forms.

All field areas which are sampled during monitoring activities shall be analyzed by laboratories and data shall be utilized for characterization waste disposal. Actual protocol for field sampling may be found in the Field Sampling and Analysis Plan for the Remedial Investigation of Waste Area Grouping 2 at Oak Ridge National Laboratory, Oak Ridge, Tennessee (ORNL 1991).

9. Organization or person responsible for performing analysis on waste samples for characterization purposes:

Internal and external laboratories contracted through the Analytical Project Office will perform all analyses on environmental samples or waste samples for waste characterization.

10. What analysis will be requested to characterize waste?

Water samples are analyzed for contaminants including but not limited to: radionuclides (H-3, Sr-90, CO-60, Cs-137, gross alpha, gross beta, and transuranics- $\mathrm{Am}, \mathrm{Pu}, \mathrm{Cm}$, Th, $\mathrm{U})$, volatile organics, semi-volatile organics, and metals $(\mathrm{Ag}, \mathrm{Al}, \mathrm{B}, \mathrm{Ba}, \mathrm{Be}, \mathrm{Ca}, \mathrm{Co}, \mathrm{Cr}$, $\mathrm{Cu}, \mathrm{Fe}, \mathrm{K}, \mathrm{Mg}, \mathrm{Mn}, \mathrm{Mo}, \mathrm{Na}, \mathrm{Ni}, \mathrm{Sb}, \mathrm{Si}, \mathrm{Sr}, \mathrm{Tl}, \mathrm{V}, \mathrm{Zn}, \mathrm{As}, \mathrm{Cd}, \mathrm{Pb}, \mathrm{Se}$, and $\mathrm{Hg}$ ).

Sediment samples have been analyzed using a Toxicity Characterization Leaching Procedure (TCLP) in order to analyze for metals, volatiles, semi-volatiles, PCBs and Pesticides. Sediments and soils are also analyzed for radionuclides (Sr-90, Tc-99, gamma, and transuranics). 
11. List the process knowledge available to characterize the waste:

The above analyses (\#10) have been performed on environmental samples collected within WAG 2 and shall be used for waste characterization. Data may be found in Tables 2, 3, and 4 of the Project Waste Management Plan.

12. Is the available process knowledge enough to properly characterize the waste without having to perform analysis on the waste?

NO (However, if existent analytical knowledge of samples placed in waste stream is sufficient to properly characterize waste produced by the WAG 2 project, then documented processed knowledge will be used.) 
13.

Estimated Types and Volume of Waste

\begin{tabular}{|c|c|c|c|c|c|}
\hline TASK & $\begin{array}{l}\text { WASTE } \\
\text { STREAM/SOURCE }\end{array}$ & TYPE & CATEGORYC & $\begin{array}{l}\text { VOLUME } \\
\text { (Annual) }\end{array}$ & $\begin{array}{l}\text { SUSPECTED } \\
\text { CONTAMINANTS }\end{array}$ \\
\hline \multirow[t]{4}{*}{$\begin{array}{l}\text { Seeps and } \\
\text { Tributanes } \\
\text { (Water sampling) }\end{array}$} & Residual Samples & Liquid & Radıological & $87 \mathrm{ft}^{3}$ & $\begin{array}{l}\text { H-3, Sr-90, Co-60, } \\
\text { Cs-137, } \\
\text { U-238/234/232, Cm- } \\
243\end{array}$ \\
\hline & Laboratory Analysis & Luquid & $\begin{array}{l}\text { Radiological } \\
\text { NonHazardous }\end{array}$ & $5 \mathrm{ft}^{3}$ & $\begin{array}{l}\mathrm{H}-3, \mathrm{Sr}-90, \mathrm{Co}-60, \\
\mathrm{Cs}-137, \\
\mathrm{U}-238 / 234 / 232, \mathrm{Cm}- \\
243, \mathrm{KMnO}_{4}\end{array}$ \\
\hline & & & Radıologıcal & $11 \mathrm{ft}^{3}$ & $\mathrm{H}-3$ \\
\hline & $\begin{array}{l}\text { Decontamination, } \\
\text { Personal Protective } \\
\text { Equipment (PPE), } \\
\text { sample containers }\end{array}$ & $\begin{array}{l}\text { Plastic, Paper, } \\
\text { Glass }\end{array}$ & Radiological & $197 \mathrm{ft}^{3}$ & Sr-90, Co-60, Cs-137 \\
\hline \multirow{7}{*}{$\begin{array}{l}\text { Hydrologic } \\
\text { Pathways } \\
\text { (Groundwater } \\
\text { sampling) }\end{array}$} & $\begin{array}{l}\text { Well Dnlling } \\
\text { Decontamination }\end{array}$ & Liquid & Radiological & $67 \mathrm{ft}^{3}$ & $\begin{array}{l}\mathrm{H}-3, \mathrm{Sr}-90, \text { Co-60, } \\
\text { Cs- } 137\end{array}$ \\
\hline & Drilling & Sludge/Sorl & Radıological & $29 \mathrm{ft}^{3}$ & Sr-90, Co-60, Cs-137 \\
\hline & $\begin{array}{l}\text { Decontamination, } \\
\text { PPE }\end{array}$ & $\begin{array}{l}\text { Plastic, Paper, } \\
\text { Glass }\end{array}$ & Radiological & $10 \mathrm{ft}^{3}$ & $\begin{array}{l}\text { Sr-90, Co-60, } \\
\text { Cs-137 }\end{array}$ \\
\hline & $\begin{array}{l}\text { Water Samplıng } \\
\text { Resıdual samples }\end{array}$ & Liquid & Radiological & $35 \mathrm{ft}^{3}$ & $\begin{array}{l}\mathrm{H}-3, \mathrm{Sr}-90, \mathrm{Co}-60 \\
\mathrm{Cs}-137\end{array}$ \\
\hline & Laboratory Analysis & & $\begin{array}{l}\text { Radıologıcal } \\
\text { Nonhazardous }\end{array}$ & $.35 \mathrm{ft}^{3}$ & $\begin{array}{l}\mathrm{H}-3, \mathrm{Sr}-90, \mathrm{Co}-60 \\
\mathrm{Cs}-137, \mathrm{KMnO}_{4}\end{array}$ \\
\hline & & & Radiological & $7 \mathrm{ft}^{3}$ & $\mathrm{H}-3$ \\
\hline & $\begin{array}{l}\text { Decontamination, } \\
\text { PPE }\end{array}$ & $\begin{array}{l}\text { Plastic, Paper, } \\
\text { Glass }\end{array}$ & Radiological & $6 \mathrm{ft}^{3}$ & $\begin{array}{l}\text { Sr-90, Co-60, } \\
\text { Cs-137 }\end{array}$ \\
\hline \multirow{3}{*}{$\begin{array}{l}\text { Sediment } \\
\text { Transport } \\
\text { (Storm-water } \\
\text { Sampling) }\end{array}$} & $\begin{array}{l}\text { Water Samplıng } \\
\text { Residual samples }\end{array}$ & $\begin{array}{l}\text { Liquid } \\
\text { (stormwater) }\end{array}$ & Radiological & $551 \mathrm{ft}^{3}$ & $\begin{array}{l}\mathrm{H}-3, \mathrm{Sr}-90, \mathrm{Co}-60 \\
\mathrm{Cs}-137\end{array}$ \\
\hline & Decontamination & Liquid & Radıological & $28.8 \mathrm{ft}^{3}$ & $\begin{array}{l}\mathrm{H}-3, \mathrm{Sr}-90, \mathrm{Co}-60 \\
\mathrm{Cs}-137\end{array}$ \\
\hline & $\begin{array}{l}\text { Decontamination, } \\
\text { PPE }\end{array}$ & $\begin{array}{l}\text { Plastıc, Paper, } \\
\text { Glass, Aluminum }\end{array}$ & Radiological & $28.9 \mathrm{ft}^{3}$ & Cs-137, Co-60, Sr-90 \\
\hline $\begin{array}{l}\text { Hydraulıc Head } \\
\text { Monitonng } \\
\text { Stations (HHMS) }\end{array}$ & $\begin{array}{l}\text { Well Purging and } \\
\text { Water Sampling }\end{array}$ & $\begin{array}{l}\text { Liquid (possible } \\
\mathrm{pH}>12 \text { ) }\end{array}$ & Radiological & $86.1 \mathrm{ft}^{3}$ & $\begin{array}{l}\mathrm{H}-3, \mathrm{Sr}-90, \mathrm{Co}-60 \\
\mathrm{Cs}-137\end{array}$ \\
\hline \multirow[t]{3}{*}{$\begin{array}{l}\text { Floodplain } \\
\text { Soil/Sediment }\end{array}$} & $\begin{array}{l}\text { Soll and Sedıment } \\
\text { Core Residual } \\
\text { Samples }\end{array}$ & $\begin{array}{l}\text { Soll and } \\
\text { Sedrment }\end{array}$ & Radiological & $2 \mathrm{ft}^{3}$ & $\begin{array}{l}\text { Sr-90, Co-60, } \\
\text { Cs-137, } \\
\text { Pu-239/240 }\end{array}$ \\
\hline & $\begin{array}{l}\text { Decontamination, } \\
\text { PPE }\end{array}$ & $\begin{array}{l}\text { Plastic, Paper, } \\
\text { PVC piping, } \\
\text { Glass and Wood }\end{array}$ & Radiological & $98 \mathrm{ft}^{3}$ & $\begin{array}{l}\text { Sr-90, Co-60, } \\
\text { Cs-137, } \\
\text { Pu-239/240 }\end{array}$ \\
\hline & Decontamination & Liquid & Radiological & $232 \mathrm{ft}^{3}$ & $\begin{array}{l}\mathrm{Sr}-90, \mathrm{Co}-60 \\
\mathrm{Cs}-137, \mathrm{Pu}-239 / 240\end{array}$ \\
\hline
\end{tabular}




\begin{tabular}{|c|c|c|c|c|c|}
\hline TASK & $\begin{array}{l}\text { WASTE } \\
\text { STREAM/SOURCE }\end{array}$ & TYPE & CATEGORYC & $\begin{array}{l}\text { VOLUME } \\
\text { (Annual) }\end{array}$ & $\begin{array}{l}\text { SUSPECIED } \\
\text { CONTAMINANTS }\end{array}$ \\
\hline $\begin{array}{l}\text { Tributary } \\
\text { Assessment } \\
\text { Task }\end{array}$ & Residual samples & Liquid & Radiological & $24.4 \mathrm{ft}^{3}$ & $\begin{array}{l}\text { H-3, Sr-90, Co-60, } \\
\text { Cs-137, } \\
\text { U-238/234/232 }\end{array}$ \\
\hline \multirow[t]{3}{*}{ (Water Sampling) } & Laboratory Analysis & & $\begin{array}{l}\text { Radiological } \\
\text { Nonhazardous }\end{array}$ & $.6 \mathrm{ft}^{3}$ & $\begin{array}{l}\mathrm{H}-3, \mathrm{Sr}-90, \mathrm{Co}-60 \text {, } \\
\mathrm{Cs}-137 \\
\mathrm{U}-238 / 234 / 232 \\
\mathrm{KMnO}_{4}\end{array}$ \\
\hline & & & Radiological & $1.2 \mathrm{ft}^{3}$ & $\mathrm{H}-3$ \\
\hline & $\begin{array}{l}\text { Decontamination, } \\
\text { PPE, sample } \\
\text { containers }\end{array}$ & $\begin{array}{l}\text { Plastic, Paper, } \\
\text { Glass }\end{array}$ & Radiological & $35.5 \mathrm{ft}^{3}$ & $\begin{array}{l}\text { Sr-90, Co-60, } \\
\text { Cs-137, } \\
\text { U-238/234/232 }\end{array}$ \\
\hline \multirow[t]{2}{*}{ Ground Water } & $\begin{array}{l}\text { Redevelopment of } \\
\text { wells }\end{array}$ & Liquid & Radiological & $100.3 \mathrm{ft}^{3}$ & $\begin{array}{l}\mathrm{H}-3, \mathrm{Sr}-90, \mathrm{Co}-60 \\
\mathrm{Cs}-137\end{array}$ \\
\hline & & $\begin{array}{l}\text { Plastic, Paper, } \\
\text { Glass }\end{array}$ & Radiological & $60 \mathrm{ft}^{3}$ & Sr-90, Co-60, Cs-137 \\
\hline
\end{tabular}

a Coring, drilling, deconning, surface/ground water sampling, protective equipment, well urging, etc.

b Liquid, metal, plastic, sediment, sludge, soil, etc.

c Hazardous, mixed, nonhazardous, radiological, TRU, TSCA, etc.

d ER Methodology for generating waste volume estimates is to be used in estimating waste volumes. Unless if you have an approved alternate methodology for estimating waste volume.

What Methodology was used to estimate your waste volumes?

Methodology used for estimation of waste volumes for each task was based on previous sampling experience.

Hydraulic Head Monitoring Stations (HHMS)- Well purging :

Volume of borehole $=\quad(\underline{D})^{2} \mathrm{~h}$

$\mathrm{D}=$ diameter of borehole

$\mathrm{h}=$ depth of borehole

Waste $=$ (volume of borehole) $\mathrm{x}$ (\# of boreholes)

volume of borehole $=28.7 \mathrm{ft}^{3}$

\# boreholes = 3 


\section{Surface Soil/Sediment sampling:}

Soil/sediment is obtained from hand augers (sampling approximately up to $2 \mathrm{ft}$ ). Most of the soil collected shall be used for sample analysis. Small waste volumes are produced from residual sample. However, the volumes listed in \# 13 were calculated as follows:

Soil/Sediment Waste $=[($ volume of core $)(\#$ cores $)] \times .25$

(75\% of soil collected will be shipped to analytical laboratories for analysis.)

Decontamination Liquid Waste $=(\#$ cores $) \times(3.5 \mathrm{~L}$ waste $/$ core $)$

(3.5 L of liquid waste has been produced in the past, including decontamination of core and all stainless steel equipment.)

volume of core $=.04 \mathrm{ft}^{3}$

\# cores $=188$

\section{Seeps and Tributaries:}

Liquid waste $=$ (volume of residual samples) $\mathrm{x}$ (\# samples)

Liquid waste (Laboratory analysis) $=$

$\left(\mathrm{KMnO}_{4}\right)=(50 \mathrm{~mL}$ waste/sample $) \times$ (\# samples)

$(\mathrm{H}-3)=(100 \mathrm{~mL}$ waste $/$ sample $) \times($ \# samples $)$

Compactible solid waste $=$

$\left[\left(2 \mathrm{ft}^{3} /\right.\right.$ sampling event $) \times$ (sampling events) $]+[(\#$ sample containers $) \times$ (volume of each container)]

\# of samples: 300

sampling events: 3

\# sample containers: 1200 total (varying in size)

volume of each container: $(1 \mathrm{~L})=.0353 \mathrm{ft}^{3},(20 \mathrm{~mL})=.00071 \mathrm{ft}^{3},(250 \mathrm{~mL})=.0088 \mathrm{ft}^{3}$

\section{Hydrologic Pathways:}

Well Drilling:

Sludge/Soil waste $=($ volume of well $) \times(\#$ of wells $)$

Compactible solid waste $=\left(5 \mathrm{ft}^{3}\right.$ waste/well $) \times(\#$ of wells $)$

Decontamination Liquid waste $=\left(3.34 \mathrm{ft}^{3}\right.$ waste/well $) \times$ (\# of wells $)$

\# of wells: 2

volume of well: $1.47 \mathrm{ft}^{3}$ 
Water Sampling:

Liquid waste $=$ (volume of residual sample) $\mathrm{x}$ (\# of samples)

Liquid waste (laboratory analysis)

$\left(\mathrm{KMnO}_{4}\right)=(50 \mathrm{~mL} / \mathrm{sample}) \times$ (\# of samples)

$(\mathrm{H}-3)=(100 \mathrm{~mL} / \mathrm{sample}) \times(\#$ of samples $)$

Compactible solid waste $=\left(1 \mathrm{ft}^{3}\right.$ waste/sampling event $) \times(\#$ of events $)$

volume of residual sample $=50 \mathrm{~mL}$

\# of samples $=200$

\# of events $=6$

\section{Tributary Assessment Task:}

Liquid waste $=[($ volume of residual sample $) \times$ (\# of samples $)]+[(3 \mathrm{~L}$ waste/ISCO tub $) \times(\#$ ISCO tubs)]

Liquid waste (laboratory analysis) $=$ $\left(\mathrm{KMnO}_{4}\right)=(50 \mathrm{~mL} / \mathrm{sample}) \times$ (\# of samples)

$(\mathrm{H}-3)=(100 \mathrm{~mL} / \mathrm{sample}) \times$ (\# of samples)

Compactible solid waste $=\left[\left(1 / 2 \mathrm{ft}^{3}\right.\right.$ waste/sampling event) $\mathrm{x}$ (\# of sampling events) $]+[(\#$ sample containers) $\mathrm{x}$ (volume of each container)]

volume of residual sample $=1.05 \mathrm{~L}$

\# of samples $=\mathbf{3 5 0}$

\# ISCO tubs $=108$

\# sampling events $=6$

\# sample containers $=2100$ total (varying in size)

volume of each container: $(1 \mathrm{~L})=.0353 \mathrm{ft}^{3},(20 \mathrm{~mL})=.00071 \mathrm{ft}^{3},(250 \mathrm{~mL})=.0088 \mathrm{ft}^{3},(100$ $\mathrm{mL})=.00355 \mathrm{ft}^{3}$

\section{Sediment Transport:}

Liquid waste (storm water) $=$ (\# storm events) $\times\{[(15$ ISCO tubs/storm) $\times$ ( $24 \mathrm{~L}$ waste/tub) $]+[(120 \mathrm{~L}$ waste (manual sampling)/storm) $]\}$

Decontamination Liquid waste $=[(15$ ISCO tubs/storm) $\times$ (3L waste/tub) $\times$ (\# storms) $]+[(\#$ sample containers and equipment) $\mathrm{x}$ (volume of waste produced for each container cleaning)]

\# storm events $=4$ (Variable)

\# sample containers and equipment for 1 year : volume of waste produced for each container cleaning $=$ 
1,920 sample containers : $250 \mathrm{~mL}$ waste produced for each container 200 filtration apparatuses : $500 \mathrm{~mL}$ waste produced for each

50 bottom withdrawal tubes : $1.1 \mathrm{~L}$ waste produced for each

Compactible waste $=\left[\left(3.21 \mathrm{ft}^{3}\right.\right.$ field waste/event $)+\left(4.01 \mathrm{ft}^{3}\right.$ lab waste/event $\left.)\right] \mathrm{x}$ (\# storm events)

\section{Ground Water Task:}

Liquid Waste $=\left(6.68 \mathrm{ft}^{3}\right.$ waste/well $) \times(\#$ of wells $)$

Compactible solid waste $=\left(4 \mathrm{ft}^{3}\right.$ waste/well $) \times(\#$ of wells $)$

\# of wells $=15$

14. Will a spill control kit be on-site during waste generation activities? YES

What will the spill control kit consist of?

A spill control kit at a minimum will contain an adequate supply of absorbent pads, chemical resistant gloves, shovel and a broom. The spill control kit container has large lettering on the outside of the container that spells out "spill control kit".

Is a first responder emergency action plan in place, in case of spill or a line puncture? This means in addition to contacting Laboratory Protection (911).

First responder emergency protocol may be found in Section 7.3 of the Project Waste Management Plan or the Health and Safety Plan for the Remedial Investigation of Waste Area Grouping 2 at Oak Ridge National Laboratory, Oak Ridge, Tennessee (Cofer et.al. 1993).

15. What organization or person will be assigned the responsibility of properly containerizing the waste, labeling the waste containers, and labeling and bagging contaminated material?

This responsibility lies with WAG 2 waste generators and laboratory stewards maintaining waste in collection areas. The Health and Safety Officer or ES\&H site oversight responsibility is to adhere to ORNL SWSA-6 WAC in addition to health physics manual.

16. Which of the following areas will be required to temporarily store the project waste until the waste can be transferred to ORNL Waste Management Operations?

\section{A. Radiological Waste Storage Area: YES}


Location: Laboratories 311 and 227 / Building 1505

Responsible organization or person that will manage areas:

Bonnie B. Lu

B. 90-Day Storage Facility for hazardous or mixed waste: NO

C. Satellite Accumulation Area: YES

Location: Laboratories 311 and 227

Responsible organization or person who will manage area:

Bonnie B. Lu

D. What is in place to protect the structural integrity and weatherability of drums and B-25 boxes.

Within Wag 5, waste filled drums will be stored on a platform and covered with plastic. Waste will be disposed of in a timely manner.

E. If any of the above areas will not be used to manage waste, please give specific details to how this waste will be managed.

Examples: stockpiling, solidification, incineration, synthetic or clay linings, in situ vitrification, leave waste in area of contamination, etc.

A 90-Day Storage Facility will not be utilized. Mixed or Hazardous waste will be collected in the Satellite Accumulation Areas which is limited to a maximum volume of 55 gallons of HAZARDOUS Waste, of which 1 quart may be acutely HAZARDOUS Waste ( $P$ listed waste or a $K, F$ or $U$ listed waste with a $(H)$ Hazard Code.

Have training requirements (e.g., Satellite Accumulation Area and 90-Day Accumulation Area) been fulfilled to manage the storage area? YES

17. Have you spoken to ORNL compliance personnel pertaining to your requirements for managing a temporary satellite, 90-day or radiological waste storage area?

David Skipper

Marilyn Hendricks (ESD)

Persons Contacted $\underline{576-5748}$

574-7296

List the Specific Requirements:

Satellite Accumulation Area:

- The satellite accumulation area shall be outlined with red tape.

- Sign shall be posted reading Satellite Accumulation Area 
- At a minimum, all RCRA requirements will be met.

- Containers of hazardous waste must be labeled properly in the field and in the laboratory with a label which is LEGIBLE and VISIBLE on the CLOSED PRIMARY container. Each label must contain the words "Hazardous Waste or Hazardous Waste, Mixed Waste" and the PRINTED NAMES of the Chemical Constituents (Formulas optional). It is recommended the concentration of chemical constituents be included on the label (if known or best estimate).

- Containers of hazardous waste must be in good condition (not leaking or damaged) and compatible with the waste being stored in them.

- Containers of hazardous waste must be kept closed unless waste is being added or removed. For solid wastes, a bag that is tied shut is sufficient. For liquid wastes, the lid must prevent a release if the container overturned.

- Containers of liquid hazardous waste should be secondarily contained to minimize the possibility of a spill. This secondary containment shall hold 10 percent of the total volume of the liquid waste stored in the area, or the volume of the largest container, whichever is larger.

- Training Requirements: Initial satellite accumulation training will be mandatory for all personnel generating hazardous waste. Retraining or successful completion of a "proficiency exam" will be required annually.

18A. Can the ORNL Hazardous Waste Group take charge of the hazardous or mixed waste generated? YES

\section{List any specific requirements:}

Requirements as stated in \# 17.

Volume of Waste (cubic feet): $0 \mathrm{ft}^{3}$

K.G. Edgemon $\underline{574-1779}$

Marilyn Hendricks (ESD) $\quad$ 574-7296

Persons Contacted

Phone

18B. Can the Solid radioactive Waste group accept the volume of radiological waste that will be generated? YES

List any specific requirements:

- Low level Compactible waste must be double bagged in radioactive materials bags.

- Waste must be free of liquid.

- Waste must be properly labeled, and characterized. 
- Suspected radionuclides and estimation of concentration should be included on label.

- Waste must meet acceptance criteria.

Volume of Waste (cubic feet): $171.79 \mathrm{ft}^{3}$

$\frac{\text { Greg Larson }}{\text { Person Contacted }} \quad \frac{1-3273}{\text { Phone }}$

18C. Can the Liquid Gaseous Waste Group accept the liquid waste that will be generated? YES

List any specific requirements:

- Waste containers must be labeled properly in the field and in the laboratory with a label which is LEGIBLE and VISIBLE on the CLOSED PRIMARY container. Each label should contain the words "Radioactive Waste" or "Hazardous Waste " as appropriate, and the PRINTED NAMES of the Constituents (Formulas optional). It is recommended the concentration of radionuclide constituents be included on the label (if known or best estimate).

- Waste must meet the acceptance criteria for Process Waste Treatment Plant or Low Level Liquid Waste System.

- Waste containers must be appropriately tagged by Health Physics.

- Appropriate disposal forms, signatures and waste certification must be completed.

Maintenance requirements for waste in the Radioactive Waste Collection Areas:

- Waste containers must be in good condition (not leaking or damaged) and compatible with the waste being stored in them.

- Waste containers must be kept closed unless waste is being added or removed. The lid must prevent a release if the container overturned.

- Waste containers should be secondarily contained to minimize the possibility of a spill. This secondary containment shall hold 10 percent of the total volume of the liquid waste stored in the area, or the volume of the largest container, whichever is larger.

Volume of Waste (cubic feet): $338.1 \mathrm{ft}^{3}$

$\frac{\text { Chris Scott }}{\text { Person Contacted }} \quad \frac{574-7057}{\text { Phone }}$

18D. Do you plan to generate any brine solution, sludge or sediment waste, if so how will you manage this waste? 
Sediment and soil waste shall be dewatered and disposed of appropriately through ORNL Waste Operations Group.

18E. Will any large volumes of uncontaminated spoils be generated? NO

18F. For waste that cannot be managed by any of the above ORNL Waste Operation Groups, what is your alternative for managing the waste?

All waste generated can be managed by the above ORNL Waste Operation Groups.

19. Will any TRU/or TRU Mixed Waste be generated? NO

20. What organization or person will be responsible for transporting the waste from the generation area to the temporary waste storage area to await final pickup by Martin Marietta Energy Systems (MMES)?

WAG 2 field personnel shall transport waste from the generation area to the temporary waste storage area within Building 1505 or designated areas. Regulated hazardous substances will not be transported on public roads.

The hydrologic pathway task will temporarily store waste at the generation area within WAG 5.

21. Will ORNL Waste Management Operations be responsible for transporting waste from site of waste generation or the waste storage area to a MMES storage, treatment or disposal facility? ORNL Waste Operation does not normally pick up waste from off-site.

ORNL Waste Management Operations transports waste from the temporary waste collection areas in the laboratory or the WAG 5 waste storage area as appropriate to the Process Waste Treatment Plant (PWTP), Non-Radiological Waste Water Treatment Plant (NRWTP), Low Level Liquid Waste (LLLW) system, or to silos or Tumulus Units in SWSA 6.

22. For waste that is generated off-site, who or what organization will transport that waste to ORNL Waste Operations? Give specific details as to how this waste will be transferred to ORNL Waste Operations?

Any waste which will be generated off-site will be transported to Building 1505 by WAG 2 project personnel. 
23. A. Will waste require transporting over public roads? YES

Waste which shall be transported over public roads is not considered RCRA Hazardous or TSCA materials. Waste generated will not exceed the DOT regulatory limit of .002 $\mu \mathrm{Ci} / \mathrm{mL}$ for radioactive materials.

B. Will waste require transporting over DOE roads before 9 a.m. and after 4 p.m.? YES

C. List the Department of Transportation issues that are of concern in transporting this waste:

none

D. Have you communicated those concerns of transporting the waste and associated resolutions to ORNL Transportation Operation Management Department and received their concurrence of how the waste may be transported? YES

Greg Livengood $\underline{574-9458}$

Person Contacted Phone

E. List the resolutions to those concerns:

Reaffirmation of compliance with all DOT regulations.

24. Identify your waste minimization techniques in that will be implemented during this task

Waste Minimization practices are addressed in Section 6.0 of the Project Waste Management Plan. 


\section{APPENDIX B}



WASTE TTEM DENTUTCATION

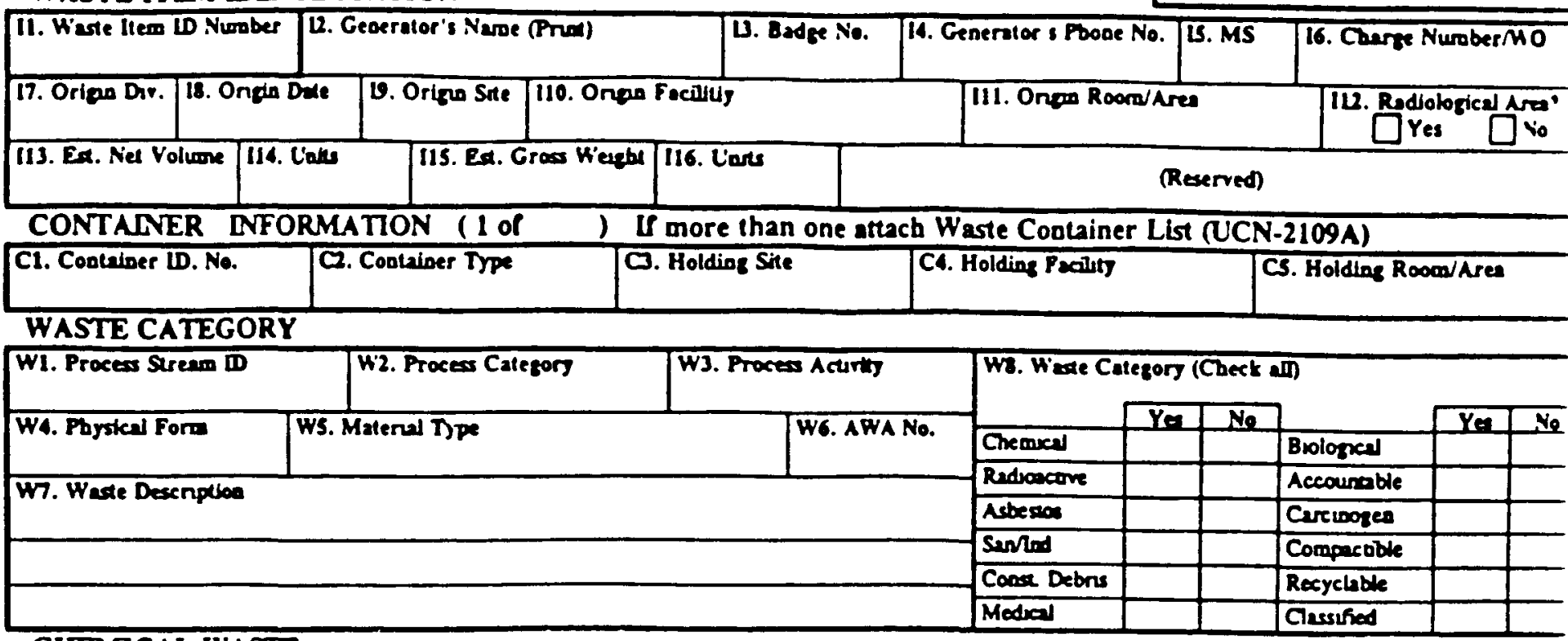

\section{CHEMICAL WASTE}

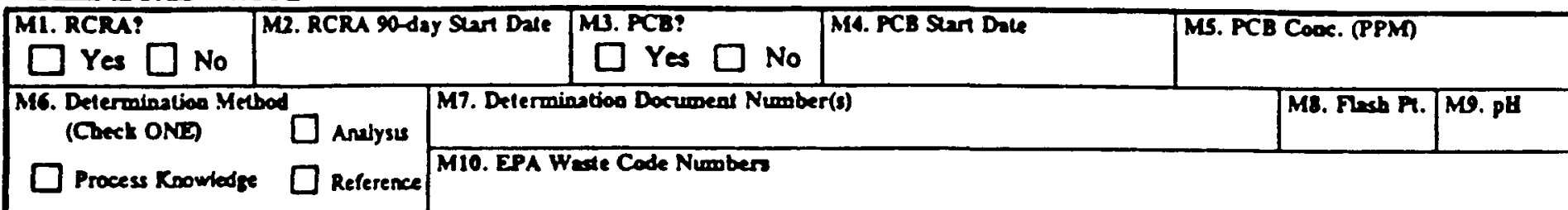

\begin{tabular}{|l|l|l|l|l|l|}
\hline MIL. Subrance ID & M12. Vol \% \\
\hline & \\
\hline & \\
\hline & \\
\hline & \\
\hline
\end{tabular}

Mi3. Substance Name

RADIOACTIVE WASTE

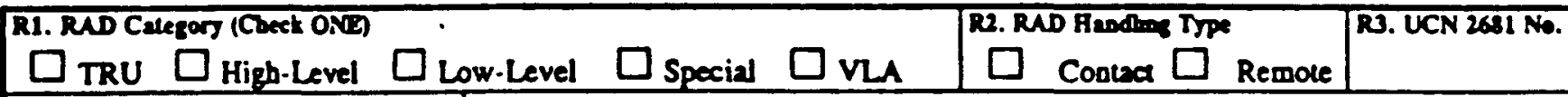
R4. Detereninetion Mletbed (Cbeck ONA) Q Anolyes $\square$ Process Knowledge $\square$ Refereves RS. Dedermintion Document Numbert(s) R6. Cbembel Pors (Reserved)

\begin{tabular}{|c|c|c|c|c|c|c|c|c|}
\hline R7. Isotope & R8. Ex. Q1y & R9. Onfts & R7. Esolope & R2. Est. Q6 & R9. Units & R7. Isotope & Re. Rx. Q48 & R9. Units \\
\hline & & & & & & & & \\
\hline & & & & & & & & \\
\hline & & & & & & & & \\
\hline
\end{tabular}

\section{HANDLDNG DFORMATION}

\begin{tabular}{|c|c|c|c|}
\hline \multicolumn{3}{|c|}{ H1. Haodling Instruction } & $\begin{array}{l}\text { B4. EP Tas } \\
\text { Color }\end{array}$ \\
\hline & & & Alph \\
\hline F2. Repinter & & H3. Cartridge DPe & Bethanm \\
\hline Noes & Half & & Nevitos \\
\hline
\end{tabular}

HL. Piclup Sie

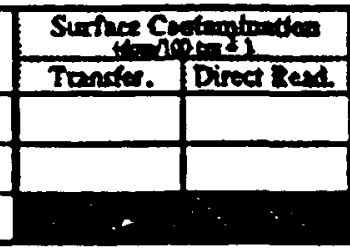

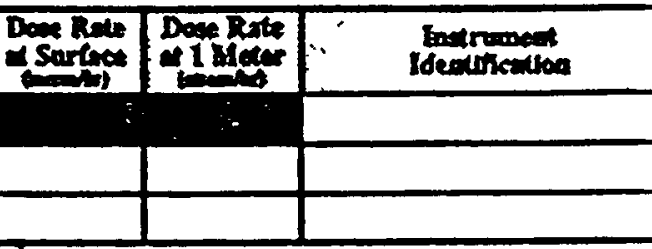

197. Fichup Area

SIGNATURES and APPROVALS

\begin{tabular}{|c|c|c|}
\hline S1. Geverwar & Bedpe & Deste \\
\hline 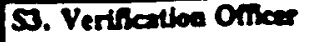 & Badge & Dene \\
\hline
\end{tabular}

\begin{tabular}{|c|c|}
\hline S. IP Technledas & Bedre \\
\hline S. Dentrathe Creasiba & Bedre \\
\hline
\end{tabular}


MARTIN MARIETTA

\section{WASTE ITEM CONTINUATION}

(Continuation Page 2 of 2 )

\begin{tabular}{|c|l|l|}
\hline $\begin{array}{c}\text { Sequence } \\
\text { Number }\end{array}$ & \multicolumn{2}{|c|}{ Referenced from WDD (UCN-2109) } \\
\cline { 2 - 3 } 1 & I1. Waste Item ID Number & C1. Container DD. No. \\
\hline
\end{tabular}

\section{CHEMICAL WASTE}

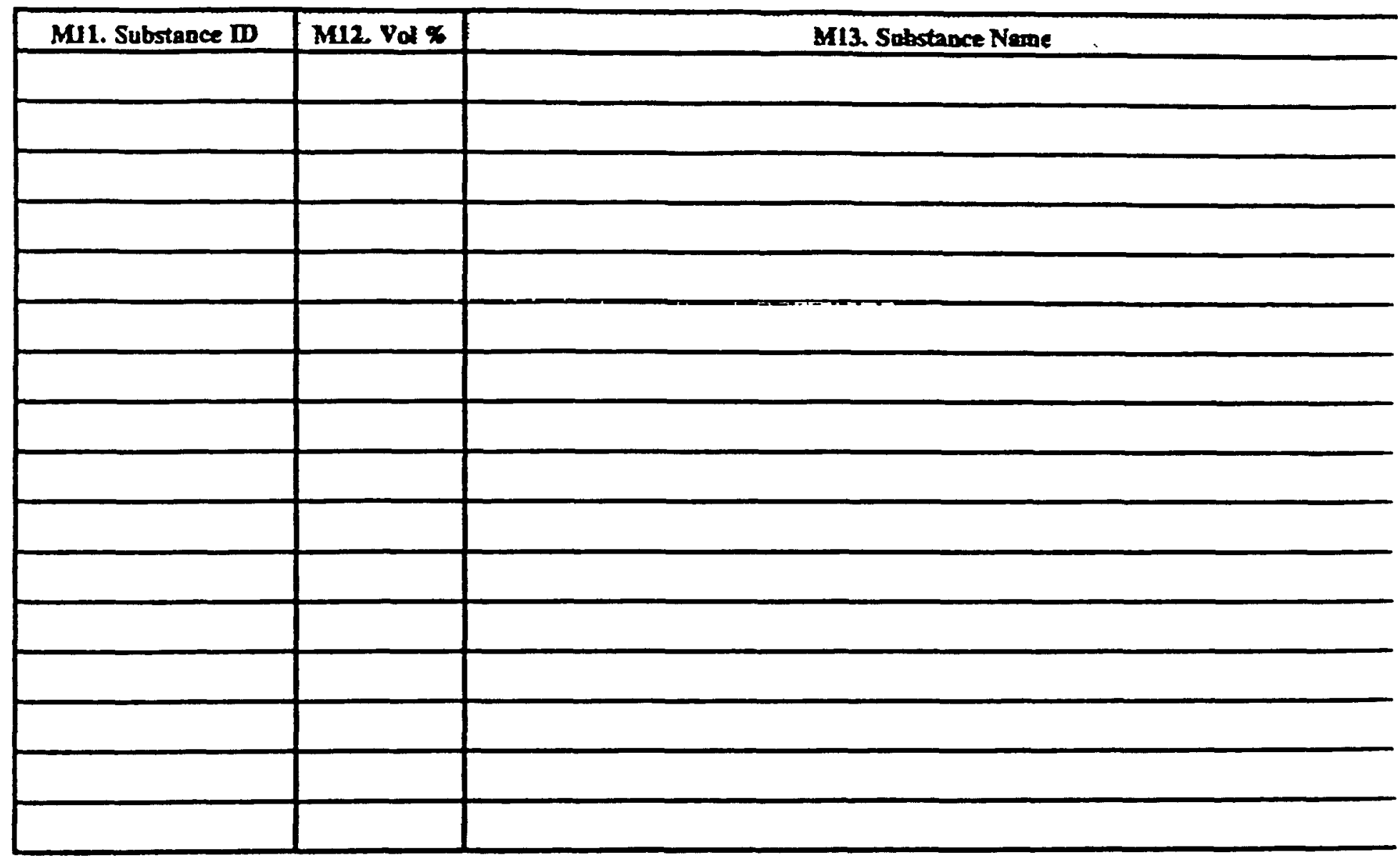

RADIOACTTVE WASTE

\begin{tabular}{|c|c|c|c|c|c|c|c|c|}
\hline R7. Isotope & RA. Rs. OtY & B9. Units & R2. Lolope & R日. Bd. Qt & B9. Unias & R7. Isolepe & R8. Rx OAY & R9. Units \\
\hline & & & & & & & & \\
\hline & & & & & & & & \\
\hline & & & & & & & & \\
\hline
\end{tabular}


MARTIN MARIETTA

\section{CONTAINER PACKING LIST}

\section{CONTADNER IDENTIFTCATION}

\begin{tabular}{|l|l|}
\hline C1. Cootainer W. No. & Cl. Contuber Type \\
\hline
\end{tabular}

C3. Holding Ske

CONTAINER PACKING LIST

\begin{tabular}{|c|c|c|c|}
\hline 11. Weste Item ID Nomber & I1. Waste Item D Number & 11. Waste Ilem In Number & 11. Warte liem In Namber \\
\hline & & & \\
\hline & & & \\
\hline & & & \\
\hline & & & \\
\hline & & & \\
\hline & & & \\
\hline & & & \\
\hline & & & \\
\hline & & & \\
\hline & & & \\
\hline & & & \\
\hline & & & \\
\hline & & & \\
\hline & & & \\
\hline & & & \\
\hline & & & \\
\hline & & & \\
\hline & & & \\
\hline & & & \\
\hline & & & \\
\hline & & & \\
\hline & & & \\
\hline & & & \\
\hline & & & \\
\hline & & & \\
\hline & & & \\
\hline & & & \\
\hline & & & \\
\hline
\end{tabular}

HANDLING INFORMATION

\begin{tabular}{|c|c|c|c|c|c|c|}
\hline \multicolumn{2}{|l|}{ H1. Heodling Instructions } & H4. HP Tas & 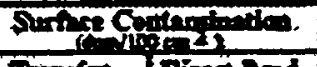 & Donesis & Degre & helromean \\
\hline & & Appha & & & & \\
\hline \multirow{2}{*}{$\begin{array}{l}\text { 16. Reopirator } \\
\square \text { None } \square\end{array}$} & \multirow{2}{*}{ 13. Cartoldse Type } & Bew/Gamma & & & & \\
\hline & & Nevisos & & & & \\
\hline \multicolumn{7}{|c|}{ SIGNATURES and APPROVALS } \\
\hline Si. Certification Oncer & Bedpo & Dene & \multicolumn{2}{|l|}{ S2. HP Techolden } & Iedor & Dece \\
\hline 53. WMO Oncer & Bedere & Dene & \multicolumn{2}{|l|}{ SA. } & Bedpe & Date \\
\hline
\end{tabular}


MarTIN MARHETTA

WASTE PICKUP REQUEST

\section{REOUEST}

P1. Requea Number

P5. Request Date

P10. Comments
P3. Badge No.

P8. Pickup Room/Area
P4. Requentor's Pbone No.
P7 Pickup Facility
P6. Pickup Stte

\section{PICKUP LIST}

\section{Winte Item er Countiver $\mathrm{BB}$}

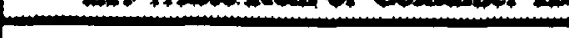

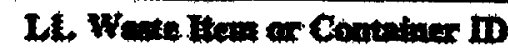

11. Waste Itum or Contriner $D$

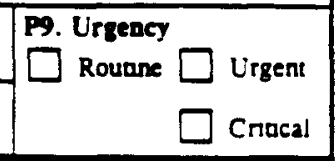

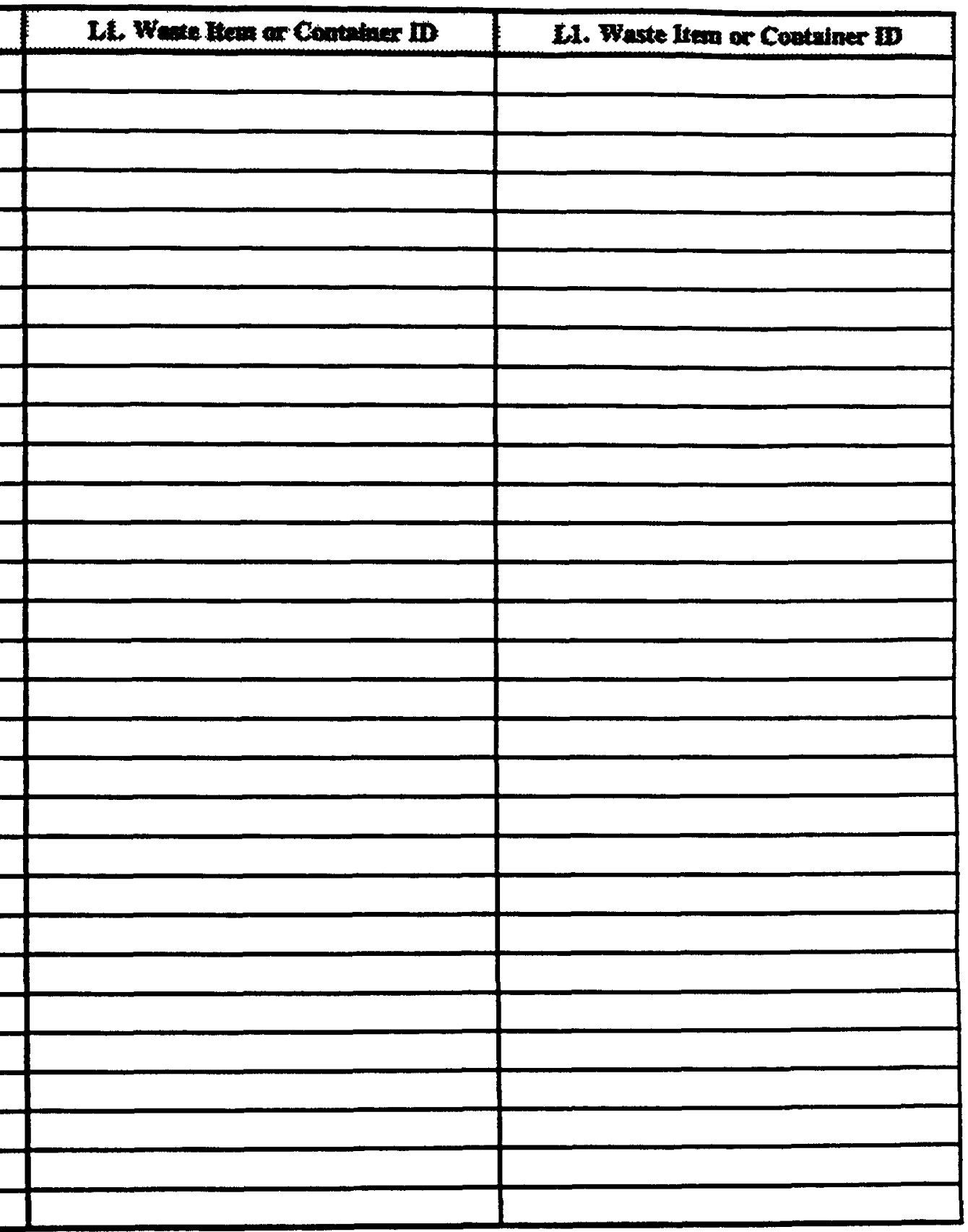

SIGNATURE and APPROVALS

\begin{tabular}{|l|l|l|}
\hline S1. Requedor & Bedre & Dete \\
\hline S3. WMO Oncer & Bedre & Dare \\
\hline
\end{tabular}

5.

S4.

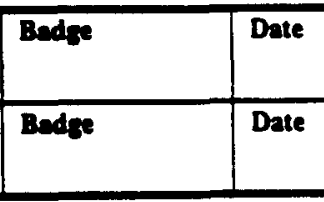


Appendix C 


\section{Waste Item Description (TX-5745) Form User Instructions}

PURPOSE: The Waste Item Description (WID) form lists the identity and the charactenstics of a waste item, the smallest trackable unit of waste. It also lists the identity of the shipping/storage container into which the waste tem may have been placed, and any applicable handing information. All of the information on the form is required in order to register the waste item with the site waste management organization, however some of the information is only applicable to specific waste categones.

\section{WASTE ITEM IDENTIFICATION}

Form ID Number/Waste Item Barcode Label (upper nght comer):

Each waste item is identified by attaching the pre-prnted waste ttem ID barcode label to it. A duplicate barcode label is attached to the WID form that describes the waste item in the rectangular box located in the upper nght comer of the form

11. Waste Item ID Number (Compulsory)

Copy the ID number of the waste ttem from the barcode label atached to the waste ttem.

Example: X1019300001

12. Generator's Name (Compulsory)

PRINT the name of the individual responsible for the generation of the waste tem.

13. Badge No. (Compulsory)

The badge number of the waste generator.

Example: 99999

14. Generator's Phone No. (Compulsory)

The complete phone number where the waste generator can be reached.

Exampie: (999) 999-9999

15. MS (Compulsory)

The mail stop of the waste generator.

Format: 9999

16. Charge Number/wO (Compulsory)

A valid charge account or work order number for waste handling charges.

Format: $9999-9999$ or A9999AAA

17. Origin Div. (Compulsory)

The numencal division code of the generating division.

Example: 27

18. Origin Date (Compulsory)

The date that the waste item was generated or declared waste.

Example: 4/1/93

19. Origin Site (Compulsory)

The site (plant) where the waste was generated.

Examples: X10. Y12, K25

110. Origin Facility (Compulsory)

The facility (building) where the waste was generated.

Example: 4500N

I11. Origin Room/Area (Compulsory)

The room or area where the waste was generated.

Example: 125L

112. Radiological Area? (Compulsory)

Is the Origin Room/Area a radiological area (yes or no)? 
113 Est Net Volume (Compulsorv)

The estumated net volume of the waste item

Format number

114 Vol Units (Compulsory)

The estumated net volume units

Values CUIN, CUFT CUYD, CC, M3, OZ, PT, QT, GAL, ML, L

115 Est Gross Weight (Compulsory)

The estumated gross weight of the waste tem

Format number

116 Wt Units (Compulsory)

The estimated net weight units

Values OZ, LB, TON, MG, G, KG

\section{CONTAINER INFORMATION}

If the waste item is placed in a standard shipping/storage contaner, the container will have it's own unique container ID barcode label attached to it The contauner ID number on the contauner barcode label is undependent of the waste uem ID number on the waste item barcode label

Waste generation activities often include the accumulation of waste in a location different from the generation location When this is the case. the accumulation location is referred to as the 'holding' location Complete the holdung location information if applicable

C1 Contaner ID Number (Compulsory)

The unque ID number of the standard shipping/storage contauner for this waste tem copied from the container barcode label

Example X10C9300123

C2 Contaner Type (Compulsory)

The container type code of the outer container of the waste item. Select the most detauled container type code avaulable from the following list If there is no sutuble contauner type code or the waste tem is self-contaned, enter 'SELF' and provide any additional detailed packaging information in the WASTE DESCRIPTION

Refer to the following page 
DRLMS

DRUM6D55

DRUM6D30

DRUM6DIS

DRUM6DS

DRUM17C55

DRUM17C30

DRUM17C10

DRUMI7CS

DRUMITESS

DRUMI7E30

DRUMI7E10

DRUMITES

DRUM17E!

DRUM17H30

DRUMITHSS

DRUM17HAS5

DRUMSSSS

DRUMSS30

DRUMBL55

DRUMBL3O

DRUMS110

DRUMS96

DRUMS85

DRUMS80

DRUMSSS

DRUMS35

DRUMS30

DRUMS2S

DRUMS

DRUMF55

DRUMF40

DRUMF30

DRUMF2S

DRUMF10

DRUMF5

DRUMF

DRUMP

DRUM

CANS

CANMI

CANM2

CANMS

CANMI

CANM20

CASKS

CASKC4

CASKC6

CASKC12

\section{Descriotion}

Drum, DOT 6D, Sieel.

Poly-lined, $55 \mathrm{gal}$

Drum, DOT 6D, Steel,

Poly-lined. $30 \mathrm{gal}$

Drum, DOT 6D. Siecl.

Poly-lined. $15 \mathrm{gal}$

Drum, DOT 6D, Sieel,

Poly-lined, 5 gal

Drum, DOT 17C, Sieel. 55 gal

Drum. DOT 17C, Steel, $30 \mathrm{gel}$

Drum, DOT 17C. Steel. 10 gal

Drum. DOT 17C. Steel. 5 gal

Drum. DOT 17E. Steel. 55 gal

Drum. DOT 17E. Steel, $30 \mathrm{gal}$

Drum, DOT 17E. Steel, $10 \mathrm{gal}$

Drum, DOT 17E. Steel, 5 gal

Drum. DOT 17E. Steel. I gal

Drum, DOT 17H. Steel, $30 \mathrm{gal}$

Drum. DOT 17H, Steel, 55 gal

Drum. DOT 17H-A. Suinless

steel. $55 \mathrm{gel}$

Drum. Suninless Steel. 55 gal

Drum. Slainiess Sleel. $30 \mathrm{gal}$

Drum. Black Iron. 55 gal

Drum. Black Iron. $30 \mathrm{gel}$

Drum. Steel. 110 gal

Drum. Steel, 96 gal

Drum, Steel, 85 gel

Drum. Steel, 80 gal

Dnum. Steel, 55 gal

Drum. Steel, 35 gal

Drum. Steel, $30 \mathrm{gal}$

Drum. Sleel, $25 \mathrm{gal}$

Drum. Sleel

Drum. Fiber, $55 \mathrm{gal}$

Drum. Fiber. 40 gal

Drum. Fiber. $30 \mathrm{gal}$

Drum. Fiber, $25 \mathrm{gal}$

Drum. Fiber. $10 \mathrm{gal}$

Drum, Fiber, 5 gal

Drum. Fiber

Drum. Polyethylene

Drum

\section{Descriotion}

Can. Metal, I gal

Can. Metul, 2 gal

Can, Metal, 5 gal

Can, Metal, $10 \mathrm{gal}$

Can, Metal, $20 \mathrm{gal}$

\section{Dexcription}

Cank, Concrele, 4.5\% wall

Cask, Concrete, $6^{\circ}$ wall

Cask. Concrete, $12^{\circ}$ wall
BOXES

BOXB25

BOX8X8

BOX $4 \times 4$

BOXM

BOXW

BOX F

BOX

TANKS

TANKP600

TANKP330

TANKP

TANKM

TANK

DLMPSTERS

DUMPSTER

ROLLBIN

TRUCKS

COMPTRUCK

DUMPTRUCK

TANKER

TRUCK

MISCELLANEOUS

GICAN

BAGPOLY

CARBOY

LEADCAR

CYLINDER

SELF
Descnption

Box. B-25, Meta

Box. Metul, $8 \times 8 \times 20$ feet

Box. Meul. $4 \times 4 \times 6$ feet

Box. Melal

Box. Wood

Box. Fiberboserd

Box

\section{Descriotion}

Tenk. Polyechylene, $600 \mathrm{gal}$

Tank, Polyechylene, $330 \mathrm{gal}$

Tank. Polyethylene

Tank. Meul

Tank

\section{Descriotion}

Dumpster, Steel

Roll bin

Description

Compactor truck

Dump truck

Tanker truck

Truck

\section{Description}

GI Can. 5 gal

Bag, Polyechylene, 4-mil

Carboy

Lead shielded carner

Gas cylinder

Self-contained 
C3 Holdung Site (Compulsory)

The site (plant) where the waste item/container was held dunng waste accumulation If the waste was not held in a location dunng accumulation different from the generation location enter ' $N A$ ' and omit Holding Facility (C4) and Holding Room/Area (CS)

Examples. X10, Y12, K25

C4 Holdung Faciluty (Conditional)

The faculity (bulding) where the waste tem/container was held dunng waste accumulation

Example. 4500N

C5 Holding Room/Area (Conditional)

The room/area identufier where the waste item/container was held dunng waste accumulation.

Example: $125 \mathrm{~L}$

\section{WASTE CATEGORY}

W1. Process Stream ID (Compulsory)

The identifier code of a registered process waste stream. If unknown enter "UNK"

W2. Process Category (Compulsory)

The generation process category code of the process or activity which generated this waste. Select the one that best describes the process category.

Values:
ABT - Abatement
PRO - Process/production (ongoing)
CON - Construction/Demolition
RND - Research and development
DND - Decommission/Decontamination
REM - Remediation
EXC - Excess/out-of-spec materal (ongoung)
TRE - Treatment
MNT - Mauntenance (ongoung)
UNP - One-tıme, unplanned (eg, spul)
OTP - One-tume, planned
UNK - Unknown (legacy waste only)

W3. Process Activity (Compuisory)

The generation process activity code of the process or activity which generated this waste. Select the one that best describes the process activity from the following categones. When 'OTHER' is selected for any of the categones, provide a bnef descrnption of the process activity in the WASTE DESCRIPTION field.

\section{PROCESS ACTIVITY CODES}

\author{
CLEANING AND DEGREASING \\ A01 - STRIPPING \\ A02 - ACID CLEANING \\ A03 - CAUSTIC (ALKALD CLEANING \\ AO4 - FLUSH RINSING \\ AOS - DIP RINSDNG \\ AO6 - SPRAY RINSING \\ A07 - VAPOR DEGREASING \\ AOB - PHYSICAL SCRAPING AND REMOVAL \\ A09 - CLEAN OUT PROCESS EQUTPMENT \\ A19 - OTHER CLEANDNG AND DEGREASING \\ SURFACE PREPARATION AND FTNISHING \\ A21 - PAINTING \\ A22 - ELECTROPLATING \\ A23 - ELECTROLESS PLATING \\ A24 - PHOSPHATING \\ A25 - HEAT TREATING \\ A26 - PICKLING \\ A27 - ETCHING \\ A29 - OTHER SURFACE COATING/PREPARATION \\ (SPECIFY IN COMMENTS)
}

PROCESSES OTHER THAN SURFACE PREPARATION

A31 - PRODUCT RINSING

A32 - PRODUCT FILTERING

A3 - PRODUCT DISTILLATION

A34 - PRODUCT SOLVENT EXTRACTION

A35 - BY-PRODUCT PROCESSING

A36 - SPENT CATALYST REMOVAL

A37 - SPENT PROCESS LIQUIDS REMOVAL

A38 - TANK SLUDGE REMOVAL

A39 - SLAG REMOVAL

A40- METAL FORMING

A41 - PLASTICS FORMING

A49 - OTHER PROCESSES OTHER THAN SURFACE PREPARATION (SPECIFY IN COMMENTS) 
OYE-TIME AVD IVTERMITTEYT PROCESSES

ASI - LEAK COLLECTION

AS2 - LEACHATE COLLECTION

AS3 - CLEANUP OF SPILL RESIDUES

AS4 - OIL CHANGES

ASS - FULTER/BATTERY REPLACEMENT

AS6 - DISCONTINUE USE OF PROCESS EQUIPMENT

AS7 - DISCARDING OFF-SPEC MATERIAL

AS8 - DISCARDING OUT-OF-DATE PRODUCTS OR

CHEMICALS

AS9 - LABORATORY WASTES

A60 - SLUDGE REMOVAL

A61 - CLOSURE OF WASTE OR INTERMITTENT PROCESSES

(SPECIFY DN COMMENTS)

POLLLTION CONTROL OR WASTE TREATMENT PROCESSES

A71 - FILTERING/SCREENING

A72 - METALS RECOVERY

A73 - SOLVENTS RECOVERY

A74 - INCINERATION/THERMAL TREATMENT

A75 - WASTEWATER TREATMENT

A76 - SLUDGE DEWATERING

A77 - STABILIZATION

A78 - AIR POLLUTION CONTROL DEVICES

A79 - OTHER POLLUTION CONTROL OR WASTE TREATMENT (SPECIFY IN COMMENTS)

\section{OTHER PROCESSES}

A81 - CLOTHING AND PERSONAL PROTECTIVE EQUIPMENT

A82 - ROUTINE CLEAN-UP WASTES (E G , FLOOR SWEEPINGS)

A89 - OTHER (SPECIFY IN COMMENTS)

W4 Physical Form (Compulsory)

The physical form of the waste. (Select one)

Values. SOLID, LIQUID, GAS, SLUDGE, SLURRY, EMULSION

W5 Matenal Type (Compulsory)

The material type code(s) which further describe the physical form of the waste Select all that apply within a specific physical form that best describe the waste materal A maxumum of three (3) entres are allowed At least one entry must be specified.

\author{
Phrsical Form: SOLID \\ MTO01 - ABSORBANT \\ MTOO2 - BIOLOGICAL \\ MTOO3 - CLOTH \\ MT004 - EQUIPMENT \\ MTOOS - GLASS \\ MTOO6 - GRANULAR \\ MTOO7 - METALLIC \\ MT008 - PAPER \\ MT009 - PPE (personal protective equipment) \\ MTO10 - PLASTIC \\ MTO11 - RUBBLE \\ MTO12 - SOIL \\ MTO13 - WOOD \\ MT099 - OTHER
}

\author{
Phrical Form: LIOUID \\ MT200 - GROUNDWATER \\ MT201 - OILBASED \\ MT202 - SOLVENT,HALOGENATED \\ MT203 - SOLVENT,NON-HALOGENATED \\ MT204 - WATERBASED \\ MT20S - WASTEWATER \\ MT206 - WELLWATER \\ MT299 - OTHER
}

\author{
Phrsical Form: SLUDGE \\ MT400 - BIOLOGICAL \\ MT401 - OILBASED \\ MT402 - METALLIC \\ MT403 - SOIL \\ MT404 - WATERBASED \\ MT499 - OTHER
}


MT600 - BIOLOGICAL

MT601 - OILBASED

MY602 - METALLIC

MT603 - SOIL

MT604 - SOLVENT,HALOGENATED

MT605 - SOLVENT,NON-HALOGENATED

MT606 - WATERBASED

MT699 - OTHER

\section{Physical Form: EMULSION}

MT800 - OILBASED

MT801 - WATERBASED

MT899 - OTHER

W6 AWA No. (Compulsory)

The ID number of the ORNL Asbestos Work Authonzation associated with this waste item Used for asbestos matenal only [Enter NA if not applicable.]

Format: number

W7 Waste Description (Compuisory)

A general description of the waste material and its packaging.

Examples: Oily rags un plastıc bags; Excess chemical in one liter bottle

W8. Waste Category (Compulsory)

Specific categones for the waste materal. All categones must be checked etther YES or NO to complete the form If a particular category is questionable. refer to the waste acceptance critena of the destination faculity and/or site waste management procedures.

CHEMICAL

Does the waste contuin potentially hazardous chemicals? If YES, complete the CHEMICAL WASTE section

\section{RADIOACTIVE}

Does the waste contain radioactive usotopes? If YES. complete the RADIOACTIVE WASTE section.

\section{ASBESTOS}

Does the waste contain asbestos?

\section{SAN/IND}

Is the waste suitable for sanitary/undustral land fill disposal?

\section{CONST. DEBRIS}

Is the waste 'clean' construction debrus?

MEDICAL

Did the waste onginate in a medical facility as a result of medical procedures and/or research.

BIOLOGICAL

Is the waste of biological origun (anumal carcasses, landscaping refuse, etc.)?

\section{ACCOUNTABLE}

Does the waste contain accountable radioactive materals? If YES, complete the UCN 2681 No. field (R3) with the number of the ORNL Nuclear Materal Intra-Laboratory Transfer form (UCN-2681).

\section{CARCINOGEN}

Does the waste contain known carcinogens?

\section{COMPACTIBLE}

Is the waste compactible? 


\section{REYCYCLABLE}

Is the waste potentially recyclabie?

\section{CLASSIFIED}

Is the waste classified material? NOTE: Classified waste cannot be disposed of at ORNL.

\section{CHEMICAL WASTE}

RCRA? (Required if Chemical waste)

Does the waste contain RCRA reguiated material (40 CFR 261)?

If yes, complete RCRA 90-Day Start Date (M2) when applicable

M2 RCRA 90-Day Start Date (Condutional)

The date when hazardous or mixed waste is moved from a satellite accumulation area to a 90 -day accumulation area. or the date when hazardous or mixed waste begins to accumulate in a 90-day accumulation area This field should NOT be completed with the date waste is moved to a satellite accumulation area

Example 4/1/93

M3 PCB? (Required if Chemical waste)

Is the waste PCB (greater than 2 PPM) regulated (yes or no)?

If yes, complete PCB Star Date (M4) and PCB Concentration (M5)

M4 PCB Start Date (Required if PCB waste)

The date the PCB contaminated waste $t e m$ was removed from service or declared waste

M5 PCB Concentration (PPM) (Required if PCB waste)

The PCB concentration in parts per milion (If less than "nnn", enter "nnn")

NOTE. Do not use less than or greater than values Enter the number that best charactenzes your waste

M6 Determination Method (Required if Chemical waste)

Select from the three methods the one which was used to determune the chemical charactenstics of the waste

Note The chemical and radiological charactenstics can be determined by undependent methods

PROCESS KNOWLEDGE - Documented knowledge of the generation process parameters

ANALYSIS - Laboratory analysis of samples of this waste item.

REFERENCE - Reference to other laboratory anaiysis of sumilar waste (penodic samping of a repetitive waste stream and/or batch sampling analysus)

M7 Determunation Document Numbers (Required if Chemical waste)

The ID numbers of documents (lab reports. MSDS sheets, process analysis reports, facility permits, etc) which substantiate the determination of the waste charactenstics A maximum of three (3) may be specified

Examples: MSDS8030. OAL1234

M8 Flash Pount (Conditional)

The flash pount of the waste materal in degrees $C$ when applicable.

Format. number

M9 pH (Condituonal)

The relatuve aciduly of the waste materal (pH) when applicable.

Format: number

M10 EPA Waste Code Numbers (Required if RCRA waste)

The EPA waste code numbers determuned from the waste charactenstics (RCRA waste only) A maxumum of twelve (12) may be specified. [40 CFR 261.30]

Examples: D001, U123 
M11. Substance ID (Required if RCRA waste)

The ID numbers of the chemical constituents which significantly affect the characterization, handling and management of the waste. For a pure chemical. enter its Chemical Abstract Service (CAS) number. For a commercial mixture, enter the MMES MSDS record number.

Format: $999999-99-9$ (CASH) or 999999 (MSDSW)

NOTE: If additional space is needed use the Waste Item Continuation (WIC) sheet (TX-5746A), and enter a statement notung a WIC sheet is attached [e.g. WIC (TX-5745A) attached. "I.

M12. Vol \% (Required if RCRA waste)

The volume pereentage of the substance in the waste. The value may range from 0 to 100 . A value of zero (0) indicates a trace quantity. Format: number

M13. Substance Name (Required if RCRA waste)

The common name of the chemical constituent or commercial mixture.

Examples: Ethanol, Formaldehyde. Exxon 123 Solvent

NOTE: Do not use chemical formulas or symbols.

\section{RADIOACTIVE WASTE}

R1. Rad Category (Required if Radioactive waste)

Select one radioactive waste category code:

a) TRU (Transuranic).

b) High-Level.

c) Low-Level.

d) Special (Special Case Waste), or

e) VLA (Very Low Activity).

Refer to the waste acceptance criteria of the destination facility and/or site waste management procedures for detailed information regarding radioactive waste calegories.

R2. Rad Handling Type (Required if Radioactive waste)

The radioactive waste handling code.

Values:

CONTACT - Contact handled ( $\leq 200 \mathrm{mrem} / \mathrm{hr}$ )

REMOTE - Remotely handled ( $>200 \mathrm{mrem} / \mathrm{hr}$ )

R3. UCN 2681 Number (Conditional)

The ID number of the ORNL Nuclear Material Intra-Laboratory Transfer Form (UCN-2681) form associated with this waste item. Used for Accountable Nuclear materiais only.

R4. Determination Method (Required if Radioactive waste)

Select from the three methods the one which was used to determine the radiological characteristics of the waste.

Note: The chemical and ndiological chancteristics can be determined by independent methods.

PROCESS KNOWLEDGE - Documented knowiedge of the generation process parameters.

ANALYSIS - Laboratory analysis of samples of this waste item.

REFERENCE - Reference to other laboratory andysis of similar waste (periodic sampling of a repetitive waste stream and/or batch sampling analysis).

RS. Determination Document Numbers (Required if Radioactive waste)

The ID numbers of documents (lab reports, process analysis reports, facility permits, etc.) which substantiate the determination of the waste characteristics. A maximum of three (3) may be specified.

Example: ORNL/TM-11652. LLL1234 
R6 Chemical Form (Conditional)

The chemical form of the radioactive waste tem

Specify the ONE which best describes the waste matenal. This is a partial listung oniy If additional chemical forms are needed contact your Waste Management Organization representative

BICARBONATE
BISULFATE
BISULFITE
BROMATE
BROMIDE
CARBONATE
CHLORATE
CHLORIDE
CHLORITE
CHROMATE

BICARBONATE

BISULFITE

BROMATE

CHLORATE

DICHROMATE
DIOXIDE
DISULFIDE
ELEMENTAL
FLUORIDE
HYDRIDE
HYDROXIDE
NITRATE
NITRIDE
NITRITE

DICHROMATE

DISULFIDE

ELEMENTAL

HYDRIDE

HYDROXIDE

NITRIDE

R7. Isotope ID (Required if Radioactive waste)

The ID of the radioisotopes contained in this waste tem.

Example: PU-239. U-235. AG-110M (Refer to Appendix A)
OXIDE

PERCHLORATE

PHOSPHATE

SULFATE

SULFIDE

SULFITE

TETRAOXIDE

TRIOXIDE

NOTE: If additional space is needed use the Waste Item Contunuation (WIC) sheet (TX-5746A), and enter a statement notung a WIC sheet is atrached [e.g "WIC (TX-5745A) attached."]

R8. Est. Quantity (Required if Radioactive waste)

The estumated quantity of the radioisotope in the waste. Note the corresponding UNITS of the quantity (cunes or grams) for each isotope. Specify the quantity as a decimal number or in scientific notation (Refer to Appendix A)

Examples: .0001, .3E-3

R9. Quan. Units (Required if Radiosctive waste)

The units for the radioisotope quantity.

Values: $\mathbf{G}$ (grams) or Cl (cunes) (Refer to Appendix A)

\section{HANDLING INFORMATION}

H1. Handling Instructions (Optional)

General handling instructions for the waste.

Examples: See MMES MSDS for instructions: Face shield required

H2. Respirator (Optional)

This is not completed for ORNL Generators.

H3. Cartridge (Optional)

This is not completed for ORNL Generators.

H4. HP Survey Data (completed by Heath Physies technician)

HP Tag Color:

Surface Contamination:

Dose Rate at Surface:

Dose Rate at 1 Meter:

Instrument ID:
The color code of the survey ug (G, B, Y, \& R)

The transferrable and direct reading count rate $(\mathrm{dpm} / 100 \mathrm{~cm} 2)$

The fixed contamination dose rate measured at the surface (mrem/hr)

The dose rate measured at 1 meter (mrem/hr)

The health physucs instrument ID number.

Format: A99999

H5. Pickup Site (Compulsory)

The site (plant) where the waste is to be picked up.

Examples: X10, Y12, K25 
H6 Pickup Faculty (Compulsory)

The faculty (bulding) where the waste is to be picked up

Example: $4500 \mathrm{~N}$

H7 Prckup Room/Area (Compulsory)

The room number or area identifier where the waste is to be picked up

Example. 125L

\section{SIGNATURES and APPROVALS}

\section{S1 Generator}

The signature. date and badge number of the waste generator, certifying that the information on the form is complete and accurate and that the waste is in complance with the Waste Acceptance Critena for the treatment, storage or disposal faculity to which it is being submitied

S2 H.P Technician

The signature, date and badge number of the H.P Technician certifying that the HP survey information is complete and accurate.

S3. Verification Officer

The signature, date and badge number of the GCO who certifies that the form has been properiy completed and that the waste was packaged in accordance with the treatment, storage. or disposal (TSD) facuity Waste Acceptance Cntena by individuals with the appropnate training.

S4 Denvative Classifier

This signature is not normally required at ORNL. 


\section{RADIOISOTOPES}

The following is a list of the valid isotopes. The units of measurement (curies or grams) of the estimated quantity are also provided. When the units of measurement are dependent upon whether the concentration of the isotope is less than or equal to $100 \mathrm{nCi} g$ or greater than $100 \mathrm{nCi} / \mathrm{g}$ it is 30 noted. [ $\mathrm{G}$ if $<=100 \mathrm{nCi} / \mathrm{g}$ else $\mathrm{CI}$ ]

\begin{tabular}{|c|c|c|c|c|c|}
\hline Isotope & Units & Isotons: & Uniss & Isotope & Lnits \\
\hline AC-225 & $\mathrm{Cl}$ & CD-113M & $\overline{\mathrm{Cl}}$ & $\overline{F M-255}$ & $\mathrm{Cl}$ \\
\hline $\mathrm{AC}-227$ & $\mathrm{CI}$ & $C D-115$ & $\mathrm{CI}$ & FR-221 & $\mathrm{CI}$ \\
\hline AC- 228 & $\mathrm{CI}$ & $C D-115 M$ & $\mathrm{Cl}$ & FR-223 & $\mathrm{CI}$ \\
\hline AG-108 & $\mathrm{Cl}$ & CE-131 & $\mathrm{CI}$ & GA-67 & CI \\
\hline AG-108M & $\mathrm{Cl}$ & CE-131M & $\mathrm{Cl}$ & GA-68 & $\mathrm{CI}$ \\
\hline AG-109M & $\mathrm{CI}$ & CE-139 & $\mathrm{Cl}$ & GD-152 & $\mathrm{CI}$ \\
\hline AG-110 & $\mathrm{CI}$ & CE-139M & Cl & GD-153 & $\mathrm{Cl}$ \\
\hline AG-110M & $\mathrm{Cl}$ & CE-141 & $\mathrm{Cl}$ & GE-67 & CI \\
\hline AG-111 & CI & CE-142 & CI & GE-68 & $\mathrm{CI}$ \\
\hline AG-111M & $\mathrm{Cl}$ & $C E-143$ & $\mathrm{CI}$ & H-3 & $\mathrm{Cl}$ \\
\hline AL-26 & $\mathrm{Cl}$ & CE-144 & $\mathrm{Cl}$ & HF- 175 & CI \\
\hline$A L-26 M$ & Cl & CF-249 & G* & HF-181 & $\mathrm{CI}$ \\
\hline AM-239 & $\mathrm{CI}$ & CF-250 & $\mathrm{CI}$ & HG-197 & $\mathrm{CI}$ \\
\hline$A M-240$ & CI & CF-251 & G* & HG-197M & $\mathrm{CI}$ \\
\hline AM-241 & G. & CF-252 & $\mathrm{Cl}$ & HG-203 & CI \\
\hline AM-242 & CI & CL-36 & $\mathrm{CI}$ & $1-123$ & $\mathrm{Cl}$ \\
\hline AM-242M & G* & CM-242 & $\mathrm{CI}$ & $1-125$ & $\mathrm{CI}$ \\
\hline$A M-243$ & $\mathrm{CI}$ & CM-243 & G* & $1-126$ & $\mathrm{Cl}$ \\
\hline$A M-244$ & $\mathrm{CI}$ & CM-244 & G * & $1-129$ & $\mathrm{Cl}$ \\
\hline$A M-244 M$ & CI & CM-245 & G・ & {$[-13]$} & $\mathrm{Cl}$ \\
\hline AM-245 & CI & CM-246 & $\mathrm{CI}$ & $1-132$ & $\mathrm{Cl}$ \\
\hline AM-246 & CI & CM-247 & G* & $I-132 M$ & $\mathrm{CI}$ \\
\hline AM-246M & Cl & CM-248 & Cl & I-133 & $\mathrm{CI}$ \\
\hline AR-39 & $\mathrm{CI}$ & co-s6 & Cl & I-133M & $\mathrm{CI}$ \\
\hline AS-74 & $\mathrm{Cl}$ & Co-57 & $\mathrm{Cl}$ & $1-134$ & $C I$ \\
\hline AS-76 & CI & co-s8 & $\mathrm{CI}$ & $\mathrm{I}-134 \mathrm{M}$ & CI \\
\hline AU-195 & $\mathrm{CI}$ & co-s8M & Cl & I-135 & $\mathrm{Cl}$ \\
\hline AU-195M & CI & $00-60$ & Cl & IN-111 & $\mathrm{Cl}$ \\
\hline AU-198 & CI & $\mathrm{CO}-60 \mathrm{M}$ & CI & $\mathbf{N}-111 \mathrm{M}$ & $\mathrm{Cl}$ \\
\hline AU-198M & CI & CR-51 & CI & DN-113M & $\mathrm{Cl}$ \\
\hline BA-131 & CI & CS-134 & CI & {$[N-114$} & $\mathrm{Cl}$ \\
\hline BA-131M & $\mathrm{Cl}$ & CS-134M & CI & $\mathrm{DN}-114 \mathrm{M}$ & CI \\
\hline$B A-133$ & CI & CS-135 & CI & DN-11S & $\mathrm{Cl}$ \\
\hline BA-133M & Cl & CS-135M & CI & IN-115M & CI \\
\hline BA-137M & $\mathrm{Cl}$ & CS-136 & CI & R-190 & $\mathrm{Cl}$ \\
\hline$B A-139$ & CI & CS-136M & $\mathrm{Cl}$ & R-190M & $\mathrm{Cl}$ \\
\hline BA-140 & CI & CS-137 & CI & RR-192 & CI \\
\hline BE-10 & $\mathrm{Cl}$ & CS-138 & CI & R-192M & $\mathrm{Cl}$ \\
\hline BE-7 & CI & CS-138M & CI & IR-194 & $\mathrm{CI}$ \\
\hline B1-207 & CI & CS-139 & Cl & R-194M & $\mathrm{CI}$ \\
\hline B1-208 & CI & CU-64 & Cl & $\mathrm{K}-40$ & CI \\
\hline B1-210 & CI & $\mathrm{CU}-67$ & Cl & $K-42$ & CI \\
\hline B1-210M & CI & DY-166 & CI & $K-43$ & $\mathrm{CI}$ \\
\hline BI-211 & CI & ES-253 & CI & KR-81 & $\mathrm{CI}$ \\
\hline B1-212 & CI & ES-254 & Cl & KR-8IM & $\mathrm{CI}$ \\
\hline BI-212M & CI & ES-254M & CI & $\mathbf{K R - 8 5}$ & CI \\
\hline B1-213 & CI & EU-150 & CI & KR.8SM & CI \\
\hline BI-214 & CI & EU-150M & CI & LA-138 & CI \\
\hline BK-249 & CI & EU-152 & CI & $L A-140$ & $\mathrm{Cl}$ \\
\hline$R-82$ & CI & EU-152M & CI & LU-172 & $\mathrm{Cl}$ \\
\hline BR-82M & Cl & EU-154 & CI & LU.172M & CI \\
\hline C. -14 & CI & EU-154M & Cl & LU-177 & CI \\
\hline$C A-1$ & CI & EU-15S & CI & LU-17TM & $\mathbf{C l}$ \\
\hline$C_{A}-45$ & Cl & EU-156 & Cl & MN-52 & Cl \\
\hline CD-104 & CI & EU- 160 & CI & MN-52M & $\mathrm{Cl}$ \\
\hline$C D-109$ & CI & FE-SS & Cl & MN-54 & cl \\
\hline$C D-113$ & Cl & FE-59 & Cl & MN-56 & Cl \\
\hline
\end{tabular}




\begin{tabular}{|c|c|}
\hline Isotore & Liniss \\
\hline MO-99 & CI \\
\hline NA-22 & $\mathrm{CI}$ \\
\hline NA-24 & $\mathrm{Cl}$ \\
\hline NA-2S & CI \\
\hline NB-92 & $\mathrm{CI}$ \\
\hline NB-92M & Cl \\
\hline NB-93M & $\mathrm{Cl}$ \\
\hline NB-94 & $\mathrm{Cl}$ \\
\hline NB-94M & $\mathrm{Cl}$ \\
\hline NB-95 & Cl \\
\hline NB-95M & $\mathrm{Cl}$ \\
\hline ND-144 & $\mathrm{Cl}$ \\
\hline ND-147 & $\mathrm{Cl}$ \\
\hline ND-149 & CI \\
\hline NI-56 & Cl \\
\hline NI-S9 & CI \\
\hline $\mathrm{N} 1-63$ & $\mathrm{Cl}$ \\
\hline NP-233 & Cl \\
\hline NP-235 & Cl \\
\hline NP.236 & G* \\
\hline NP-236M & $\mathrm{Cl}$ \\
\hline NP-237 & $G^{*}$ \\
\hline NP-238 & $\mathrm{Cl}$ \\
\hline NP.239 & CI \\
\hline NP-240 & $\mathrm{Cl}$ \\
\hline NP-240M & Cl \\
\hline NP-241 & $\mathrm{Cl}$ \\
\hline OS-185 & $\mathrm{Cl}$ \\
\hline OS-190M & CI \\
\hline OS-191 & CI \\
\hline OS-19IM & Cl \\
\hline OS-193 & $\mathrm{Cl}$ \\
\hline OS-194 & $\mathrm{Cl}$ \\
\hline P-32 & $\mathrm{Cl}$ \\
\hline P-33 & CI \\
\hline PA-230 & Cl \\
\hline PA-231 & Cl \\
\hline PA-232 & $\mathrm{Cl}$ \\
\hline PA-233 & $\mathrm{Cl}$ \\
\hline PA-234 & $\mathrm{Cl}$ \\
\hline PA-234M & CI \\
\hline PA-235 & Cl \\
\hline PB-196 & CI \\
\hline PE-203 & Cl \\
\hline PB-203M & $\mathrm{CI}$ \\
\hline PB-204M & CI \\
\hline PB-205 & $\mathrm{CI}$ \\
\hline PB-209 & CI \\
\hline PB-210 & CI \\
\hline PB-211 & CI \\
\hline PB-212 & CI \\
\hline PB-214 & CI \\
\hline PD-103 & CI \\
\hline PD-107 & CI \\
\hline PD-107M & CI \\
\hline PD-109 & CI \\
\hline PD-109M & CI \\
\hline PM-146 & CI \\
\hline PM-147 & Cl \\
\hline PM-148 & CI \\
\hline PM-148M & CI \\
\hline PM-149 & CI \\
\hline PO-210 & CI \\
\hline$P O-211 M$ & CI \\
\hline $\mathrm{PO}-212 \mathrm{M}$ & Cl \\
\hline PO-218 & CI \\
\hline PR-142 & CI \\
\hline PR-142M & Cl \\
\hline
\end{tabular}

\begin{tabular}{|c|c|c|c|}
\hline Isocope & Lining & Isotope & Cinits \\
\hline PR-143 & $\mathrm{Cl}$ & $S M-147$ & \\
\hline PR-144 & $\mathrm{Cl}$ & SM-148 & $\mathrm{Cl}$ \\
\hline PR-144M & $\mathrm{Cl}$ & SM-149 & $\mathrm{CI}$ \\
\hline PT-185 & $\mathrm{Cl}$ & SM-1S1 & $\mathrm{CI}$ \\
\hline PT-185M & CI & SM-1S3 & CI \\
\hline PT-191 & $\mathrm{Cl}$ & $S N-113$ & $\mathrm{Cl}$ \\
\hline PT-193 & CI & SN-113M & $\mathrm{CI}$ \\
\hline PT-193M & $\mathrm{Cl}$ & SN-117M & CI \\
\hline PT-195M & $\mathrm{Cl}$ & SN-119M & $\mathrm{Cl}$ \\
\hline PT-197 & $\mathrm{Cl}$ & $\mathrm{SN}-121$ & $\mathrm{Cl}$ \\
\hline PT-197M & $\mathrm{Cl}$ & $\mathrm{SN}-121 \mathrm{M}$ & $\mathrm{CI}$ \\
\hline PU-236 & CI & $\mathrm{SN}-123$ & $\mathrm{Cl}$ \\
\hline PU-237 & $\mathrm{CI}$ & SN-123M & $\mathrm{Cl}$ \\
\hline PU-238 & G. & SN-125 & $\mathrm{CI}$ \\
\hline PU.239 & G. & $\mathrm{SN}-125 \mathrm{M}$ & $\mathrm{Cl}$ \\
\hline PU-240 & $\mathrm{Cl}$ & SN-126 & $\mathrm{Cl}$ \\
\hline PU-241 & G* & SR-85 & $\mathrm{CI}$ \\
\hline PU-242 & $\mathrm{Cl}$ & SR-85M & $\mathrm{Cl}$ \\
\hline PU-243 & $\mathrm{Cl}$ & SR-89 & $\mathrm{CI}$ \\
\hline PU-244 & $\mathrm{Cl}$ & SR-90 & CI \\
\hline PU-245 & CI & TA-179 & CI \\
\hline PU-246 & $\mathrm{CI}$ & TA-182 & CI \\
\hline RA-222 & CI & TB-160 & $\mathrm{CI}$ \\
\hline RA-223 & $\mathrm{Cl}$ & TB-161 & $\mathrm{Cl}$ \\
\hline RA-224 & CI & TC-95 & CI \\
\hline RA-225 & CI & TC.95M & CI \\
\hline RA-226 & $\mathrm{Cl}$ & TC.99 & $\mathrm{CI}$ \\
\hline RA-228 & CI & TC.99M & $\mathrm{Cl}$ \\
\hline RA-229 & $\mathrm{Cl}$ & TE-123 & $\mathrm{Cl}$ \\
\hline RB-86 & CI & TE-123M & $\mathrm{Cl}$ \\
\hline RB-86M & $\mathrm{Cl}$ & TE-125M & CI \\
\hline RB-87 & $\mathrm{Cl}$ & TE-127 & $\mathrm{Cl}$ \\
\hline RB-88 & Cl & TE-12TM & $\mathrm{Cl}$ \\
\hline RB-89 & Cl & TE-129 & $\mathrm{CI}$ \\
\hline RB-90 & Cl & TE-129M & $\mathrm{Cl}$ \\
\hline RB-90M & CI & TE-132 & $\mathrm{CI}$ \\
\hline RE-183 & Cl & TH-226 & $G$ \\
\hline RE-186 & Cl & TH-227 & G \\
\hline RE-186M & Cl & TH-228 & G \\
\hline RE-187 & $\mathrm{Cl}$ & TH-229 & G \\
\hline RE-188 & Cl & TH-230 & G \\
\hline RE-188M & Cl & TH-231 & G \\
\hline RH-103M & $\mathrm{Cl}$ & TH-232 & G \\
\hline RH-10S & Cl & TH-233 & G \\
\hline RH-105M & Cl & TH-234 & G \\
\hline RH-106 & CI & TL-201 & CI \\
\hline RH-106M & Cl & TL-204 & CI \\
\hline RN-219 & CI & TL-206 & CI \\
\hline RN-220 & CI & TL-206M & CI \\
\hline RN-222 & CI & TL-207 & $\mathrm{CI}$ \\
\hline RU-103 & CI & TL-20TM & $\mathrm{Cl}$ \\
\hline RU-106 & Cl & TL-208 & Cl \\
\hline 5-35 & CI & TL-209 & CI \\
\hline SB- 122 & CI & $U-228$ & $\mathbf{G}$ \\
\hline SB-122M & CI & U-230 & G \\
\hline SB-124 & CI & U-231 & G \\
\hline SB-124M & CI & U-232 & G \\
\hline SB-12S & CI & U-233 & G* \\
\hline SB-126 & CI & U-234 & G \\
\hline SB-126M & CI & U-235 & G \\
\hline SB-127 & CI & U-235M & G \\
\hline Sc- 4 & Cl & U.236 & G \\
\hline SC-4M & Cl & U-237 & G \\
\hline SC-46 & Cl & U.238 & G \\
\hline SC-46M & Cl & U.239 & G \\
\hline SE-7S & Cl & U.240 & G \\
\hline SE-79 & Cl & $V-48$ & Cl \\
\hline SE-79M & Cl & $V-49$ & CI \\
\hline
\end{tabular}




\begin{tabular}{|c|c|}
\hline Isocone & Units \\
\hline V-50 & $\mathrm{Cl}$ \\
\hline$W-181$ & $\mathrm{CI}$ \\
\hline$W-187$ & $\mathrm{Cl}$ \\
\hline W-188 & $\mathrm{CI}$ \\
\hline XE-129M & CI \\
\hline XE-131M & $\mathrm{CI}$ \\
\hline XE-133 & $\mathrm{CI}$ \\
\hline$X E-133 M$ & $\mathrm{CI}$ \\
\hline$Y-88$ & CI \\
\hline Y-90 & $\mathrm{CI}$ \\
\hline$Y-90 M$ & CI \\
\hline$Y-91$ & $\mathrm{CI}$ \\
\hline Y-91M & CI \\
\hline YB-169 & CI \\
\hline YB-169M & CI \\
\hline YB-175 & CI \\
\hline $2 N-63$ & $\mathrm{CI}$ \\
\hline $2 N-65$ & CI \\
\hline ZR-93 & $\mathrm{Cl}$ \\
\hline ZR-95 & $\mathrm{CI}$ \\
\hline
\end{tabular}




\section{Container Packing List (TX-5749) Form User Instructions}

PURPOSE: The Contauner Packing List (CPL) form is used to describe a sungle standard shippung/storage container which holds multuple, separately identufied waste items. This type of packing is often referred to as 'lab-packing' It differs from a 'bulkung operation' in that the waste items, once packed, retaun their onginal identitues and charactenstics The CPL lists the type and identity of the standard shipping/storage container, the identities of all of the waste items packed unside 1 , and any applicable handiung information

Multuple, separately identified waste items which are being lab-packed in the field often ongunate from several different waste generators and locations withen the generating organization A Generator Cerification Officer is responsible for ensurng that each waste items packed in the container is identified with a properly completed WD and attached barcode label, venfying that the container is properiy sealed and labeled, and that the waste package meets all appicable site critera for transfer to the site waste management organization.

\section{CONTAINER IDENTIFICATION}

C1. Contauner ID No. (Compulsory)

The unique ID number of the shipping/storage contaner copied from the contauner barcode label

Example: X10C9300123

C2. Contauner Type (Compulsory)

The container type code of the shipping/storage container Select the most detaded container type code avaulable from the following list.

Examples: DRUMS55. DRUMS30

C3. Holding Site (Compulsory)

The site (plant) where the container was held dunng waste accumulation.

Examples: X10, Y12, K25

C4. Holding Facility (Compulsory)

The facility (building) where the waste contaner was held durng waste accumulation.

Example: $4500 \mathrm{~N}$

C5. Holding Room/Area (Compulsory)

The room/area identifier where the waste contaner was held durng waste accumulation.

Example: $125 \mathrm{~L}$

\section{CONTAINER PACKING LIST}

I1. Waste Item ID Number (Compuisory)

The unique ID number of each waste item in the contaner from the barcode labels attached to the waste items.

Example: X1019300001

\section{HANDLINGINFORMATION}

H1. Handling Instructions (Optional)

General handling unstructions for the waste.

Examples: See MMES MSDS for instructions; Face shield required

H2. Respintor

This is not completed for ORNL generators.

H3. Cartridge

This is act completed for ORNL generators. 
H4. HP Survey Data (completed by Health Physics technician) (Compulsory)

HP Tag Color:

The color code of the survey tag (G, Y, B, \& R)

Surface Contamination:

The count rate from a surface swipe $(\mathrm{dpm} / 100 \mathrm{~cm} 2)$

Dose Rate at Surface:

The fixed contamination dose rate measured at the surface (mrem/hr)

Dose Rate at 1 Meter:

The dose rate measured at 1 meter $(\mathrm{mrem} / \mathrm{hr})$

Instrument ID:

The health physics instrument ID number.

Format: A99999

\section{SIGNATURES and APPROVALS}

S1. Certification Officer (Compulsory)

The signature, date and badge number of the Generator Certification Officer, cenifying that the information on the CPL is complete and accurate, that the waste package complies with waste acceptance critena of the treatment, storage or disposal facility to which it is being submitted and that the waste was packaged by individuals with appropnate trauning.

S2. H.P. Technician (Compulsory)

The signature and badge number of the H.P. Technician certifying that the HP survey information is compiete and accurate.

S3. WMO

The signature and badge number of the Waste Management Organization (WMO) officer verifying that the CPL and all associated WID forms have been completed properly. 
APPENDIX D 


\section{ENVIRONMENTAL RESTORATION WASTE MANAGEMENT PROGRAM TOTAL GENERATED WASTE}

EONTAINEA IO MOS.

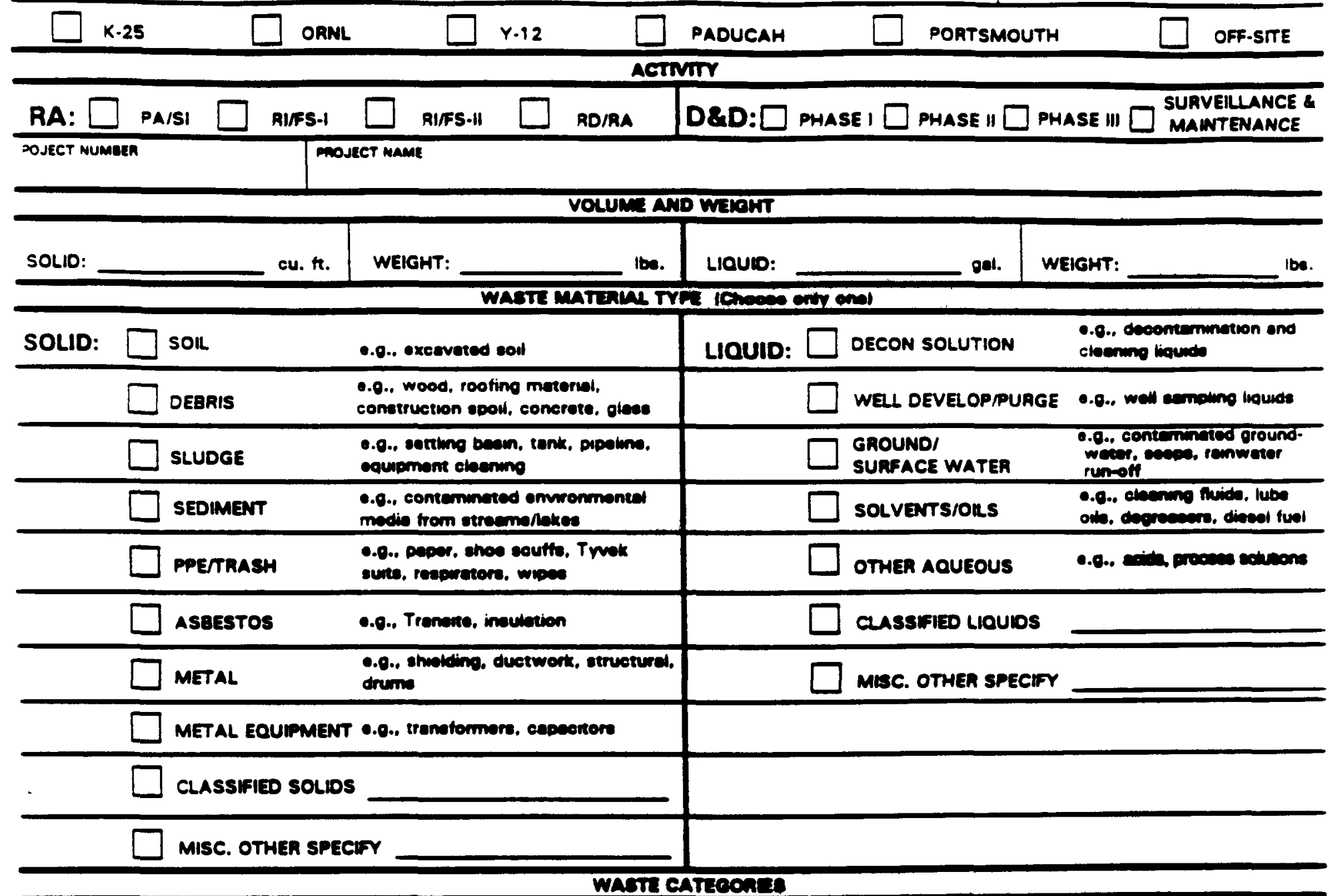

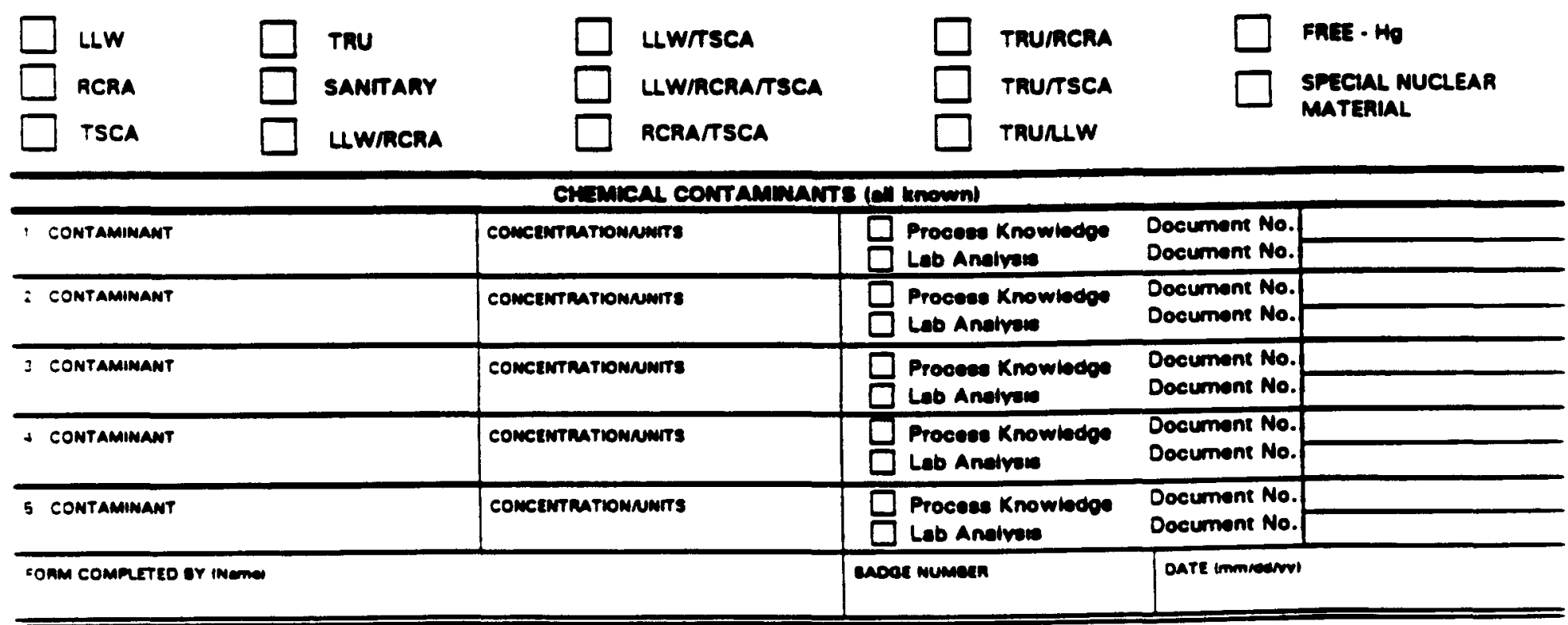




\section{DISTRIBUTION}

1. T. L. Ashwood

2-31. L. A. Baron

32. D. M. Borders

33. H. L. Boston

34. M. D. Bryant

35. B. B. Burgoa

36. P. J. Campbell

37. J. L. Carpenter

38. R. B. Clapp

39. C. Clark, Jr.

40. N. S. Dailey

41. T. L. Hatmaker

42. M. S. Hendricks

43. S. E. Herbes

44. D. S. Hicks

45. V. L. Holt

46-48. D. M. Matteo

49-50. P. T. Owen

51. J. S. Phillips

52. P. A. Schrandt

53. J. A. Shaakir-Ali

54. T. F. Scanlan

55. M. F. Tardiff

56. D. R. Watkins

57. P. S. Wood

58. B. A. Ziegler

59-61. ORNL ER Document Management Center-RC

62-64. Central ER Document Management Center

65. Laboratory Records Dept.

66. ORNL Patent Section

67. Central Research Library

68. Office of Assistant Manager for Energy Research and Development, DOE Oak Ridge Operations Office, P.O. Box 2001, Oak Ridge, TN 37831-8600

69-70. Office of Scientific and Technical Information, P.O. Box 62, Oak Ridge, TN 37831 